\title{
ESM1 promotes triple-negative breast cancer cell proliferation through activating AKT/NF-кB/Cyclin D1 pathway
}

\author{
Wentong Liu ${ }^{1}$, Yang Yang ${ }^{1}$, Bincan $\mathrm{He}^{1}$, Fengjun $\mathrm{Ma}^{1}$, Fengzeng Sun ${ }^{1}$, Min Guo ${ }^{1}$, Min Zhang ${ }^{1,2}$, \\ Zhiqiang Dong ${ }^{1,2,3}$
}

${ }^{1}$ College of Biomedicine and Health, College of Life Science and Technology, Huazhong Agricultural University, Wuhan, China; ${ }^{2}$ Hubei Cancer Hospital, Wuhan, China; ${ }^{3}$ Brain Research Institute, Taihe Hospital, Shiyan, China

Contributions: (I) Conception and design: W Liu, Z Dong; (II) Administrative support: M Guo, M Zhang, Z Dong; (III) Provision of study materials or patients: Z Dong; (IV) Collection and assembly of data: F Ma, F Sun, B He; (V) Data analysis and interpretation: W Liu, Y Yang, Z Dong; (VI) Manuscript writing: All authors; (VII) Final approval of manuscript: All authors.

Correspondence to: Zhiqiang Dong. 1 Shizishan Rd, Wuhan 430070, China. Email: dongz@mail.hzau.edu.cn.

Background: Triple-negative breast cancer (TNBC) is a subtype of invasive breast cancer that tests negative for PR, ER and excess HER2 protein. TNBC has a greater progression potential with poorer prognosis, compared with other types of breast cancer. Endothelial cell-specific molecule 1 (ESM1), also known as endocan, is overexpressed in various cancers including breast cancer and may play an important role in cancer progression.

Methods: The online resource of The Cancer Genome Atlas (TCGA) was used for analyzing the expression alteration of ESM1 in breast cancer patient tissues. We examined the changes of various malignant behaviors of TNBC cell and in vivo tumor growth after inhibiting or overexpressing ESM1 in two human TNBC cell lines, MDA-MB-468 and MDA-MB-231. When ESM1 was knocked down or overexpressed in TNBC cell, AKT and p65 phosphorylation and Cyclin D1 expression were analyzed by western blotting. The ESM1overexpressing TNBC cell was treated with MK-2206 and BAY-117082 at various concentrations.

Results: Our analyses show that ESM1 is overexpressed in TNBC cell lines as well as patient tissues, which is correlated to poor prognosis. Our results demonstrate that ESM1 knockdown decreases while overexpression of ESM1 increases in vitro proliferation, migration and invasion of TNBC cell and knockdown of ESM1 inhibits in vivo TNBC tumor growth. Our mechanistic study further discloses that ESM1 promotes the proliferation of TNBC cell through activating an Akt-dependent NF- $\mathrm{B} / \mathrm{Cyclin} \mathrm{D} 1$ pathway.

Conclusions: Our results demonstrate that ESM1 knockdown decreases while overexpression of ESM1 increases in vitro migration, proliferation and invasion of TNBC cell and knockdown of ESM1 inhibits in vivo tumor growth of TNBC in the xenograft mouse model. Our mechanistic study further discloses that ESM1 promotes the proliferation of TNBC cell through activating the Akt-dependent NF- $\mathrm{KB} /$ CyclinD1 pathway. Our findings expand the knowledge about the molecular mechanisms underlying TNBC progression and provide rationale for using ESM1 as a therapeutic target or prognostic marker for TNBC.

Keywords: Endothelial cell-specific molecule 1 (ESM1); triple-negative breast cancer (TNBC); proliferation; NF$\kappa \mathrm{B} ;$ cyclin D1

Submitted Oct 19, 2020. Accepted for publication Jan 15, 2021.

doi: $10.21037 /$ atm-20-7005

View this article at: http://dx.doi.org/10.21037/atm-20-7005 


\section{Introduction}

Breast cancer is the most common cancer and the second leading cause of cancer death in women despite the fact that deaths from breast cancer have declined in recent years benefiting from increased knowledge in the molecular mechanisms of this cancer and consequent targeted treatments (1). The subtypes of breast cancer vary greatly in many aspects such as age of onset, clinical characteristics, degree of malignancy, treatment sensitivity, and prognosis. With the development of endocrine therapy and target therapy, the five-year survival rate of patients with $\mathrm{ER}^{+}$, $\mathrm{PR}^{+}$, or HER2 ${ }^{+}$breast cancer has been greatly improved (2). For example, drugs that block estrogen receptor such as tamoxifen and treatments that lower estrogen levels such as aromatase inhibitor anastrozole can effectively inhibit $\mathrm{ER}^{+}$ breast cancer; drugs targeting HER2, such as Herceptin (chemical name: trastuzumab), have good therapeutic effects on HER2 ${ }^{+}$breast cancer. However, patients with TNBC, hardly benefit from endocrine therapy or HER2 target therapy due to a lack of drug targets. TNBC accounts for $10-15 \%$ of all newly diagnosed breast cancer and is more prone to relapse (3). Most of the triple-negative breast cancers (TNBCs) develop originally and rapidly invade at early stage (4). Systemic chemotherapy is the main method of treatment for TNBC patients after surgery, but patients are susceptible to chemotherapy resistance. However, the molecular basis that drives TNBC progression is not fully understood. Further investigation of the molecules that promote TNBC progression is of great importance for the treatment and prognosis determination of this pernicious disease.

Endothelial cell-specific molecule 1 (ESM1), also known as endocan, is a $50-\mathrm{kDa}$ secretory proteoglycan $(5,6)$. ESM1 is overexpressed in various tumors such as lung cancer (7), head and neck squamous cell carcinoma as well as ovarian cancer (8) and overexpression of ESM1 is reported to induce tumor formation through regulating cell proliferation and facilitating tumor growth (9). Previous studies have shown that ESM1 may play an important role in tumor progression through regulating angiogenesis (10). The early stage of breast cancer metastasis was correlated to increased ESM1 in murine mammary carcinoma (11). ESM1 has been considered as a potential prognostic marker of TNBC because of the facts that significantly higher expression of ESM1 was found in lung-metastasis, bone-metastasis, adrenal-metastasis and brain metastasis variants of MDA-MB-231 compared with the parental cells $(12,13)$. Furthermore, ESM1 has been found significantly overexpressed in radiotherapy-resistant MDA-MB-231 cell (14). However, the biological role of ESM1 in the malignant behaviors of TNBC cell especially the underlying molecular mechanisms remain largely unknown.

It has been reported that ESM-1 overexpression in human colorectal carcinoma cell line HCT-116 results in enhanced cell proliferation through the Akt-dependent activation of NF- $\kappa \mathrm{B}$ pathway (6). The PI3K/Akt pathway is involved in a number of cellular processes, such as quiescence, proliferation and apoptosis (15). Activation of NF- $\mathrm{KB}$ has been found to modulate the inflammation, transformation, proliferation, invasion, and chemoresistance in cancer. Irregular NF- $\kappa \mathrm{B}$ activation leads to aberrant expression of target genes such as CNND1, CNND2. Cyclin D1, encoded by oncogene CNND1, regulates the progression from the G1 to $S$ phase in the cell cycle by modulating the activity of cyclin-dependent kinases (CDKs) $(16,17)$ and is amplified in a substantial proportion of human cancers (18). Multiple signaling pathways have been demonstrated to regulate the expression of cyclin D1, including PI3K/AKT/mTOR, MAPKs, STATs, ER and NF- $\kappa$ B pathways (19).

In this study, we first analyzed the expression of ESM1 in TNBC cell lines as well as patient tissues and the correlation between ESM1 expression and the relapsefree survival time. In order to explore the role of ESM1 in TNBC progression and the underlying cellular mechanism, we adopted in vitro and in vivo studies using two TNBC cell lines. Our study discloses that ESM1 facilitates TNBC progression through enhancing the proliferation, migration and invasion of cancer cells. Further mechanistic study indicates that ESM1 promotes TNBC cell proliferation through activating an Akt-dependent NF- $\kappa \mathrm{B} / \mathrm{Cyclin} \mathrm{D} 1$ pathway. We present the following article in accordance with the ARRIVE reporting checklist (available at http:// dx.doi.org/10.21037/atm-20-7005).

\section{Methods}

All animal experiments were approved by the Scientific Ethics Committee of Huazhong Agricultural University (permit number HZAUMO-2015-016). Experiments were performed in compliance with Chinese national or institutional guidelines for the care and use of animals.

\section{Cell lines}

HBL-100 is the human breast epithelium cell line. MCF7 
is the Luminal A cell line. MDA-MB-231, HCC1937 and MDA-MB-468 were TNBC cell lines. All cell lines used in this study were purchased from ATCC, and were maintained in culture medium with $10 \%$ FBS (Invitrogen, Carlsbad, CA, USA) in the presence of $5 \% \mathrm{CO}_{2}$ at $37{ }^{\circ} \mathrm{C}$. MCF7 was maintained in DMEM and the others were maintained in RPMI.

\section{Bioinformatic analyses}

Data pertaining to ESM1 expression were retrieved from TCGA dataset (http://cancergenome.nih.gov/). Among them, 113 are normal breast tissues, and 1,070 are breast tumor tissues, as shown in total online: https:// cdn.amegroups.cn/static/public/atm-20-7005-1.pdf. Information of clinical characters including individual cancer stages, nodal metastasis status and cancer subclasses were obtained according to their respective clinical information (in total online: https://cdn.amegroups.cn/ static/public/atm-20-7005-2.pdf). The Kmplot program (http://kmplot.com/analysis/) was used for analyzing the expression of ESM1 in TNBC patient tissues.

\section{Western blotting analysis and Real-time quantitative PCR}

Western blotting and qRT-PCR were conducted as previously described (20). Primer sequences were shown in total online: https://cdn.amegroups.cn/static/public/atm20-7005-3.pdf. For western blot analysis, anti-Cyclin D1, anti-GAPDH (all mouse), goat anti-mouse or anti-rabbit antibodies were obtained from Proteintech (Chicago, IL, USA). Anti-phospho-p65, anti-p65, anti-phospho-AKT, anti-AKT (all rabbit) antibodies were purchased from Cell Signaling Technology (Danvers, MA, USA). Rabbit antiESM1 antibody was purchased from Abcam (Cambridge, MA, USA). Original blots of the Western blot were shown in Figure S1.

\section{Short bairpin RNA (shRNA) and ESM1 knockdown}

ESM1 shRNAs were designed using RNAi Designer (https://rnaidesigner.thermofisher.com). Three shRNA sequences were picked, synthesized and cloned into the pGreenPuro shRNA expression lentivector. Oligo sequences for the ESM1 shRNAs were listed in total online: https://cdn.amegroups.cn/static/public/atm-20-7005-4.pdf. HEK-293T cells were co-transfected with pGreenPuro-
ESM1shRNA or pGreenPuro, together with VSV-G and GAG-POL plasmids using Lipofectamine 3000 reagent (Thermo Scientific, USA). For ESM1 knockdown, cells were infected with the control lentivirus and those bearing ESM1 shRNAs respectively and incubated at $37^{\circ} \mathrm{C}$ for $24 \mathrm{~h}$. Cells were then selected in the culture with $2 \mu \mathrm{g} / \mathrm{mL}$ of puromycin for 3 days and then harvested for knockdown effect evaluation by qRT-PCR and western blot. The shRNA with best knockdown effect was selected and the corresponding as well as the control lentivirus was used to infect TNBC cell to establish stable shRNA-expressing cell lines through culturing the infected cells in the selection medium containing $2 \mu \mathrm{g} / \mathrm{mL}$ puromycin for 2 weeks.

\section{CCK-8 assay}

The proliferation of TNBC cell was assessed by CCK- 8 assay (Dojindo, Kumamoto, Japan) following the manufacturer's manual. Five thousand cells per well were cultured for 1,2 , and 3 days respectively at $37{ }^{\circ} \mathrm{C}$ with $5 \%$ $\mathrm{CO}_{2}$ before measurement. For CCK- 8 assay, $10 \mu \mathrm{L}$ of the CCK-8 solution were used per well and incubated for 2 hours. Cytation 5 Cell Imaging Multi-Mode Reader (BioTek Instruments, USA) was used to determine the absorbance at $450 \mathrm{~nm}$.

\section{Colony formation assay}

Cells were cultured into 6-well plates for 14 days with 200 cells per well. The colonies were then fixed with methanol. Crystal violet is used for staining. The plating efficiency is determined by counting the number of colonies and calculated as (number of colonies formed/number of cells inoculated) $\times 100 \%$.

\section{Migration and invasion assays}

Transwell chamber (BD Biosciences) with $8 \mu \mathrm{m}$ pore size were used, and $2 \times 10^{4}$ TNBC cells were cultured into the upper layer in serum-free medium, while the bottom chamber is supplied with $10 \%$ FBS. After $24 \mathrm{~h}$, cells were removed from the upper layer. The cells in the bottom chamber were stained with crystal violet and then counted under microscope. Invasion assay is performed with the same protocol as the migration assay except that the pore filter's upper side was precoated with $10 \mu \mathrm{g} / \mathrm{mL}$ Matrigel (BD Biosciences, Bedford, MA, USA). 


\section{Mouse xenograft model}

Female athymic nu/nu mice, 4-week-old with average weight of $12 \mathrm{~g}$, were purchased and raised in the Experimental Animal Center of Huazhong Agricultural University, China. Control and ESM1-shRNA1-MDA-MB-231 cells were subcutaneously injected into nude mice to create xenografted human TNBC tumor with $1 \times 10^{6}$ cells per animal and 5 injected mice each group. Tumor growth in mice was inspected every day after inoculation by measuring and calculating the tumor size by formula $\left(\mathrm{A} \times \mathrm{B}^{2}\right) / 2$, in which $\mathrm{A}$ is the largest and $\mathrm{B}$ is the smallest diameter. The mice were killed on the 42 nd day, and xenografted tumors were weighed.

\section{Inbibitor treatment}

The AKT inhibitor MK-2206 and the NF- $\mathrm{kB}$ inhibitor BAY-117082 were purchased from MCE (Monmouth, NJ, USA). Five thousand cells per well were cultured into 96well plates. MK-2206 or BAY-117082 were then applied respectively to the cultured cells at a working concentration of 1, 2, 5, and $10 \mu \mathrm{M}$, lasting for 1,2 and 3 days. For western blot analysis of protein expression, cells were seeded at $50-60 \%$ confluence followed by treatment with MK-2206 or BAY-117082 at 2 or $5 \mu \mathrm{M}$ for 1 day.

\section{Statistical analysis}

All data are presented as mean \pm SEM and analyzed by Student's $t$-test, ${ }^{*} \mathrm{P}<0.05,{ }^{* *} \mathrm{P}<0.01,{ }^{* * *} \mathrm{P}<0.001$. Experiments were performed in triplicate. GraphPad Prism v.6.01 was used for statistical analyses.

\section{Results}

\section{ESM1 is upregulated in TNBC cell lines and patient tissues}

In order to explore the role of ESM1 in TNBC progression, we first took advantage of the online resource of The Cancer Genome Atlas (TCGA) to analyze the expression alteration of ESM1 in breast cancer tissues and Kaplan-Meier plotter to explore the correlation between ESM1 expression and the relapse-free survival time of TNBC patients. Our analyses showed that ESM1 transcript level is significantly upregulated in breast cancer tissues with different stages (Figure 1A), nodal metastasis status (Figure $1 B$ ) and subtypes (Figure 1C) compared with normal tissues. We then examined ESM1 expression in breast cancer cell lines and found that ESM1 mRNA and protein expression levels were robustly increased in TNBC cell lines such as MDAMB-231, MDA-MB-468 and HCC1937, compared with the luminal breast cancer cell line MCF7 and the mammary epithelial cell line HBL-100 (Figure 1D). According to the relapse-free survival time of TNBC patients, the patients with high expression of ESM1 had poorer prognosis (Figure 1E). The high expression of ESM1 was significantly correlated with ages, advanced tumor-node-metastasis (TNM) stage and lymph metastasis in TNBC patients $(\mathrm{P}<0.05 ;$ Table 1).

\section{ESM1 knockdown decreases in vitro migration, proliferation and invasion of TNBC cell}

At cellular level, the progression of cancer is driven by the proliferation, migration and invasion of cancer cells, which result in the growth of the tumor tissue and metastasis to sites distant from the primary tumor. To study the role of ESM1 in TNBC progression and the underlying cellular mechanism, we examined the effects of ESM1 knockdown on the TNBC cells' malignant behaviors. Three short hairpin RNAs (shRNAs) targeting at different loci of ESM1 mRNA were cloned into the lentiviral vector pGreenPuro, transfected in TNBC cell (MDA-MB-231 and MDAMB-468), and tested for the knockdown effects. All three shRNAs were capable of significantly inhibiting ESM1 expression (Figure 2A,B; Figure S2). The most robust one was chosen, shRNA1 for MDA-MB-231 and shRNA3 for MDA-MB-468, and used to establish stable shRNAexpressing cell lines for further analyses. The corresponding control MDA-MB-231 and MDA-MB-468 cell lines were also created using the lentivirus bearing no shRNA sequence.

The tumor cell proliferation potential is critical for the tumor growth at both the primary and the metastatic tissues. We therefore tested TNBC cell proliferation using CCK-8 colorimetric assay and colony formation. The results of CCK- 8 assay showed that, after ESM1 expression was knocked down by shRNA, the proliferation of MDA-MB-231 cells decreased remarkably starting at $24 \mathrm{~h}$ (Figure 2C) and that of MDA-MB-468 cells decreased significantly at $48 \mathrm{~h}$ (Figure 2D) after seeding compared with corresponding control cells. We also conducted colony formation assay to further check the proliferation especially in the aspect of the clonogenic potential of MDA-MB-231, which is closely correlates with the ability to repopulate 
A Expression of ESM1 in BRCA based on individual cancer stages

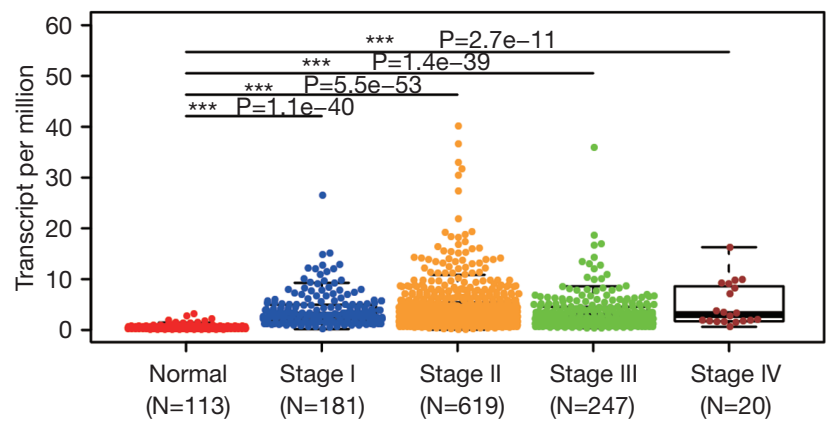

C

Expression of ESM1 in BRCA based on breast cancer subclasses

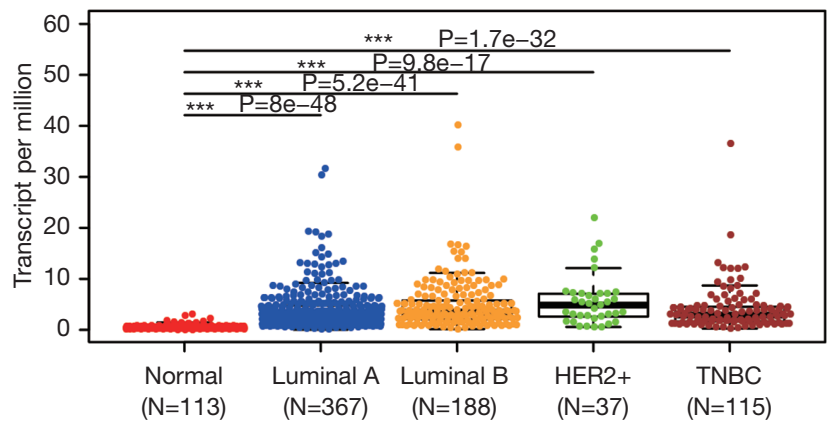

E

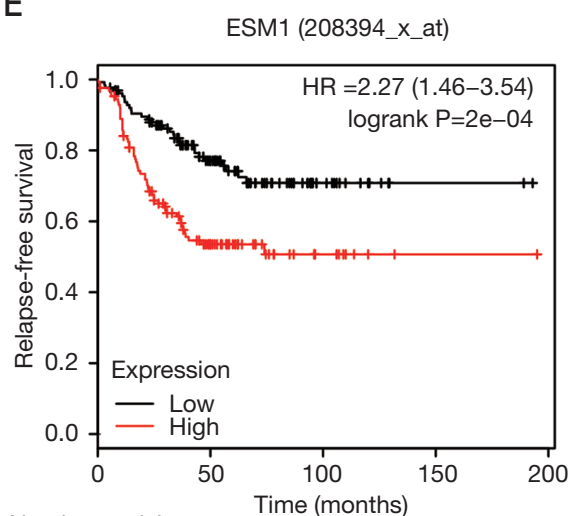

Number at risk

Low 128

High 127
B

Expression of ESM1 in BRCA based on nodal metastasis status

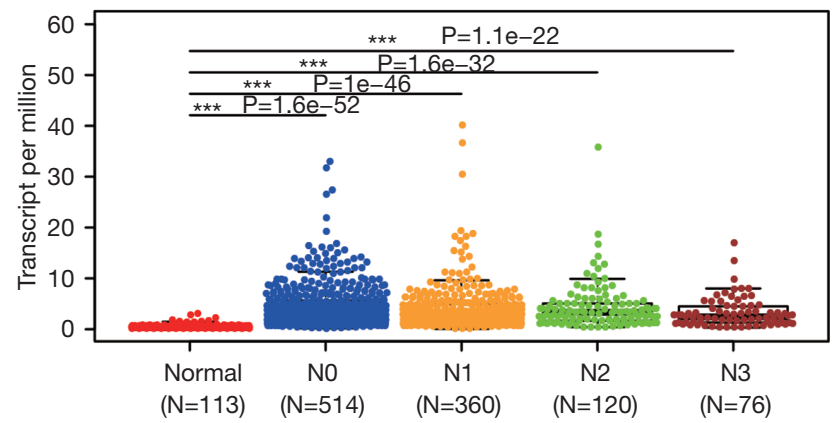

$\mathrm{D}$

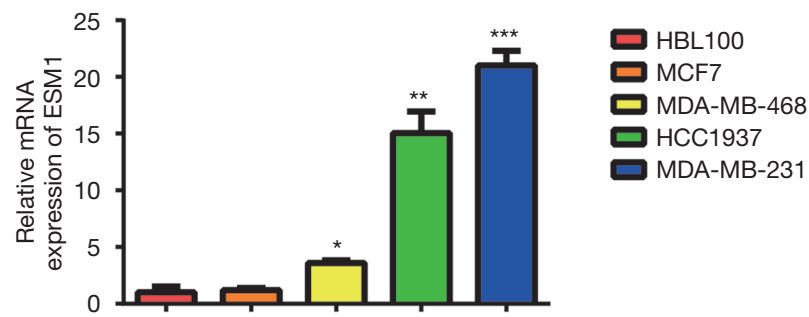

ESM1

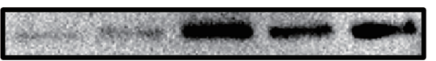

$20 \mathrm{KD}$

GAPDH

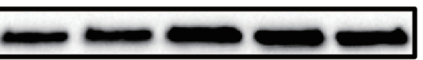

$36 \mathrm{KD}$

임

Figure 1 Expression of ESM1 is elevated in breast cancer tissues and cell lines. (A,B,C) ESM1 expression in breast cancer tissues was examined using TCGA data. (A) ESM1 expression in breast cancer tissues with different stages. (B) ESM1 expression in breast cancer tissues with different nodal metastasis status. (C) ESM1 expression in different breast cancer subtypes. (D) qRT-PCR and Western blot analysis shows the increased ESM1 expression in TNBC cell lines compared with luminal breast cancer cell line MCF7 and mammary epithelial cell line HBL-100. (E) Kaplan-Meier curves of relapse-free survival (RFS) of TNBC patients with high and low ESM1 expression. The patient number on each timepoint is listed at the bottom part. * $\mathrm{P}<0.05 ;{ }^{* *}, \mathrm{P}<0.01$; ${ }^{* *}, \mathrm{P}<0.001$. 
Table 1 The relationship between ESM1 expression and clinicopathological parameters

\begin{tabular}{|c|c|c|c|c|c|}
\hline Clinical characteristics & $\mathrm{N}$ & High expression & Low expression & $\chi^{2}$ & $\mathrm{P}$ \\
\hline Ages (years) & & & & 5.2545 & 0.02189 \\
\hline$\geq 60$ & 33 & 14 & 19 & & \\
\hline$<60$ & 78 & 42 & 36 & & \\
\hline$I+I I$ & 90 & 46 & 44 & & \\
\hline $\mathrm{III}+\mathrm{IV}$ & 21 & 10 & 11 & & \\
\hline Lymph metastasis & & & & 6.5636 & $1.04 \mathrm{E}-02$ \\
\hline No & 73 & 36 & 37 & & \\
\hline
\end{tabular}

the tumor in the metastatic site, and found out that ESM1shRNA1-MDA-MB-231 cells produced significantly less colonies than the Control-MDA-MB-231 cells (Figure 2E). Both the CCK- 8 and the colony formation assay hereto suggest an important role of ESM1 in preserving the proliferation as well as the clonogenic potential of TNBC cells.

Migration and invasion are key cellular events of tumor progression as both are crucial steps involved in the multistage process that leads to metastasis. We first examined TNBC cell migration after ESM1 knockdown using Transwell assay. The results showed that shRNA1expressing MDA-MB-231 cells demonstrated markedly less cell migration rate at $24 \mathrm{~h}$ compared to the control shRNAexpressing cells (Figure $2 F$ ). Similarly, the migration rate of MDA-MB-468 cells also decreased significantly after ESM1 was knocked down by shRNA3 (Figure $2 G$ ), indicating an essential role of ESM1 in promoting TNBC cell migration. Cancer cells need to cross the basement membrane when they initially invade lymphatic or vascular beds during dissemination. The invasion process is comprised of distinct events that include the attachment of cells to the basement membrane and the migration of cells into the target tissue. In order to study whether ESM1 plays an important role in TNBC cell invasion, we used an invasion chamber in the Transwell assays to measure the number of invasive cells migrating through precoated Matrigel, a solid gel that is used as an in vitro basement membrane, on the upper side of the pore filter. Consistent with the cell migration assay results, both shRNA1-expressing MDA-MB-231 cells (Figure $2 H$ ) and shRNA3-expressing MDA-MB-468 cells
(Figure $2 I$ ) showed a significantly decreased invasion rate compared with the respective control tumor cells, which implies that ESM1 functions to enhance the invasion potential of TNBC cell to promote progression.

Taken together, by examining and analyzing the cellular malignant behaviors of TNBC cells, MDA-MB-231 and MDA-MB-468, we showed that in vitro proliferation, migration, invasion and colony formation of TNBC cell decreased significantly when ESM1 expression was knocked down by shRNA, indicating an essential role of ESM1 in maintaining the malignancy of and consequently promoting the progression of TNBC.

\section{Inbibition of ESM1 attenuates TNBC progression in vivo}

In order to study the effect of ESM1 knockdown on the TNBC progression in vivo, ESM1-shRNA1-MDA-MB-231 and control-MDA-MB-231 cells were subcutaneously injected into nude mice to create xenografted human TNBC tumor with $1 \times 10^{6}$ cells per animal and 5 injected mice each group. Tumor growth in mice was inspected every day after inoculation by measuring and calculating the tumor size. The results showed that mice inoculated with control-MDA-MB-231 first developed measurable tumor on day 8 while ESM1-shRNA1-MDA-MB-231 mice on day 11 after inoculation and the tumor size of ESM1-shRNA1MDA-MB-231 was significantly smaller than controlMDA-MB-231 thereafter (Figure 3A, in total online: https:// cdn.amegroups.cn/static/public/atm-20-7005-3.pdf). Mice were sacrificed on day 42 and xenografted tumors were harvested and weighed. Consistent with the tumor growth 
A



MDA-MB-231

C

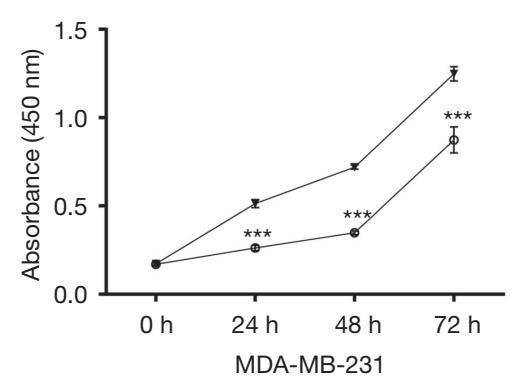

$E$

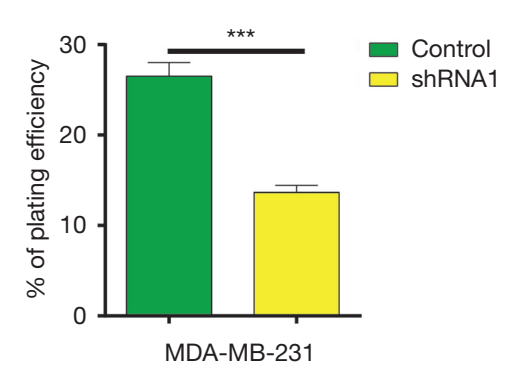

G

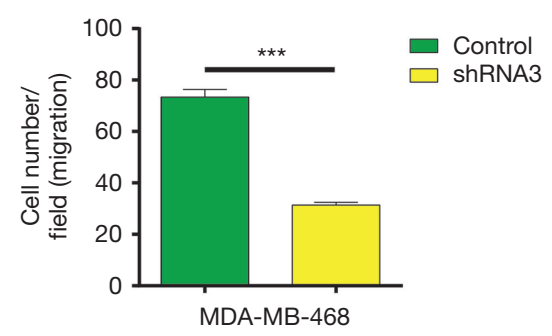

B

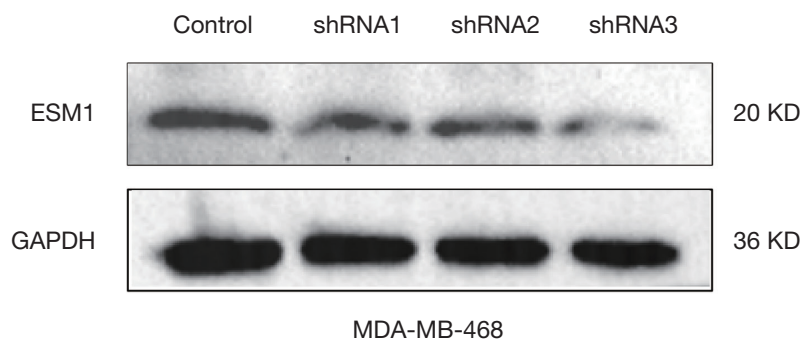

D

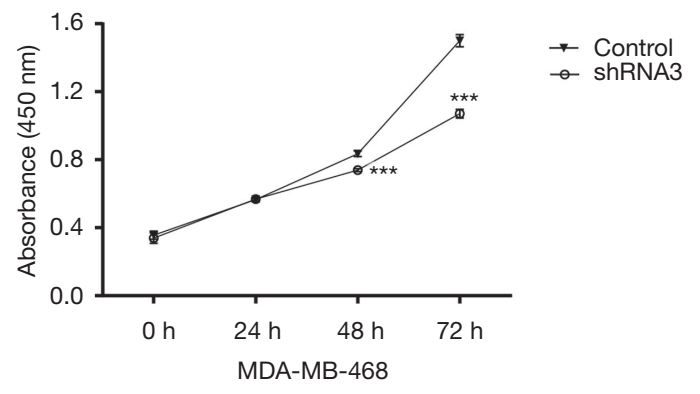

$\mathrm{F}$
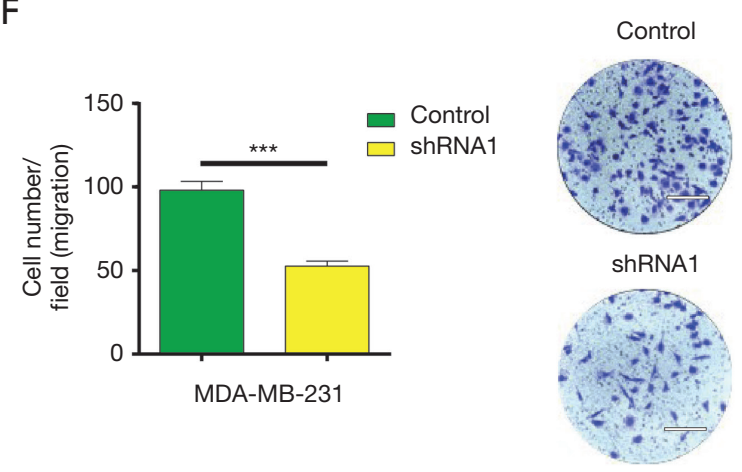

$\mathrm{H}$

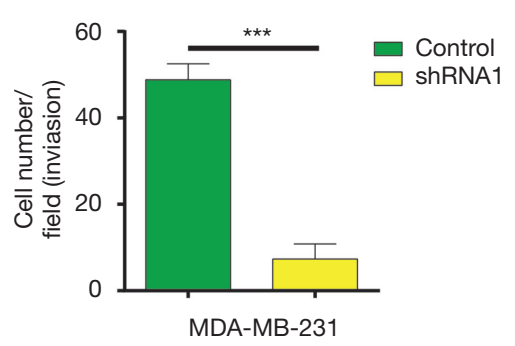

Control

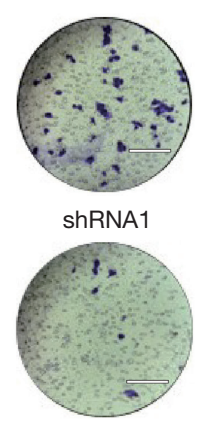



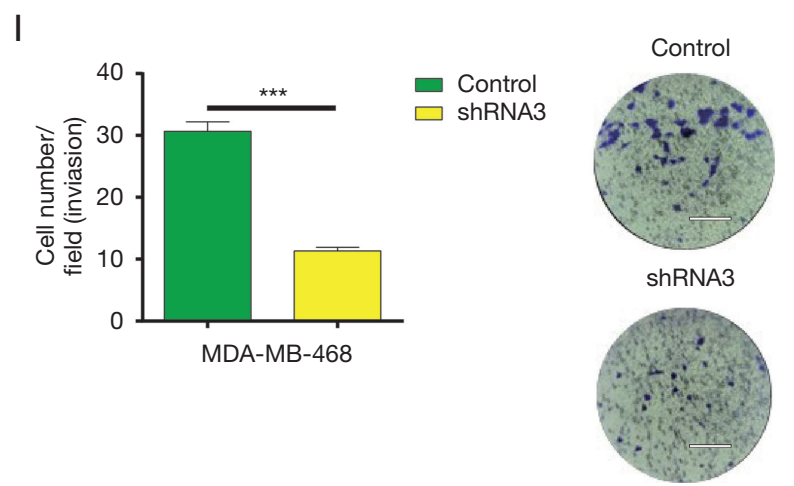

Figure 2 ESM1 knockdown decreases in vitro cell proliferation, migration and invasion in both MDA-MB-231 and MDA-MB-468 cells. (A) Western blot result shows the knockdown of ESM1 expression in MDA-MB-231 cells by three shRNAs and shRNA1 was chosen to establish stable transgenic cell line for further analyses due to its most robust knockdown effect. (B) Western blot result shows the knockdown of ESM1 protein expression in MDA-MB-468 cell by three shRNAs and shRNA3 was chosen to establish stable transgenic cell line for further analyses. (C) CCK-8 assay; (E) migration assay; (G) invasion assay; (I) colony formation assay on ESM1-shRNA1-MDAMB-231 and Control-MDA-MB-231 cells. (D) CCK-8 assay; (F) migration assay; (H) invasion assay on ESM1-shRNA3-MDA-MB-468 and Control-MDA-MB-468 cells. Scale bar, $360 \mu \mathrm{m}$. ${ }^{* * *}, \mathrm{P}<0.001$.

curve, the weight of xenografted tumors from ESM1shRNA1-MDA-MB-231 group is significantly smaller than that of control MDA-MB-231 group (Figure 3B,C). We hereby confer that ESM1 is essential to the in vivo progression of TNBC as disclosed by the fact that ESM1 knockdown results in delayed appearance and slower growth of xenografted TNBC tumor in mice.

\section{ESM1 overexpression increases in vitro migration, proliferation and invasion of TNBC cell}

Our loss-of-function study has shown that ESM1 plays a crucial role in boosting the in vitro TNBC cell potential to proliferate, migrate and invade which consequently promotes cancer progression in vivo. In order to determine whether ESM1 is sufficient to promote the cellular malignant behaviors of TNBC cell, we further performed gain-of-function experiments on cultured MDA-MB-231 and MDA-MB-468 cells. Full length cDNA sequence of ESM1 was cloned into PCDNA3 for overexpression. ESM1-PCDNA3 and PCDNA3 control plasmids were then transfected into TNBC cell by Lip3000 reagent and the overexpression of ESM1 was confirmed by western blot. We systemically examined the in vitro TNBC cell proliferation, migration, and invasion using CCK-8 analysis, colony formation analysis, and Transwell analysis respectively as described above. The results indicated that ESM1 overexpression dramatically increased TNBC cell proliferation (Figure $4 A, B, C$ ), migration (Figure $4 D, E$ ) and invasion (Figure $4 F, G$ ) in both MDA-MB-231 and MDAMB-468 cells.

\section{ESM1 promotes TNBC cell proliferation by activating

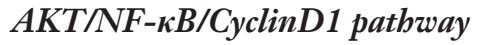

In order to study the molecular mechanism of ESM1 promoting TNBC cell proliferation, we first examined the expression of phosphorylated AKT and NF- $\mathrm{KB}$ / p65 as well as Cyclin D1 when ESM1 was overexpressed or knocked down in TNBC cell. The results showed that the phosphorylated AKT and NF- $\mathrm{NB} / \mathrm{p} 65$ were upregulated in MDA-MB-231 and MDA-MB-468 cells with overexpression of ESM1 compared with the control group, while total proteins levels of AKT and NF- $\mathrm{KB} / \mathrm{p} 65$ between the two groups remain similar. The expression of Cyclin D1 was also upregulated in MDA-MB-231 and MDA-MB-468 cells with overexpression of ESM1. On the contrary, when ESM1 was knocked down by shRNA, the levels of phosphorylated AKT and NF-kB/p65 decreased and the Cyclin D1 expression was downregulated in both MDA-MB-231 and MDA-MB-468 cells (Figure $5 A, B$ ).

Our results disclosed that overexpression of ESM1 enhances while knockdown of ESM1 suppresses phosphorylation of AKT and NF- $\mathrm{BB}$ p 65 as well as the expression of Cyclin D1 in TNBC cell. We therefore hypothesized that ESM1 promotes TNBC cell proliferation 


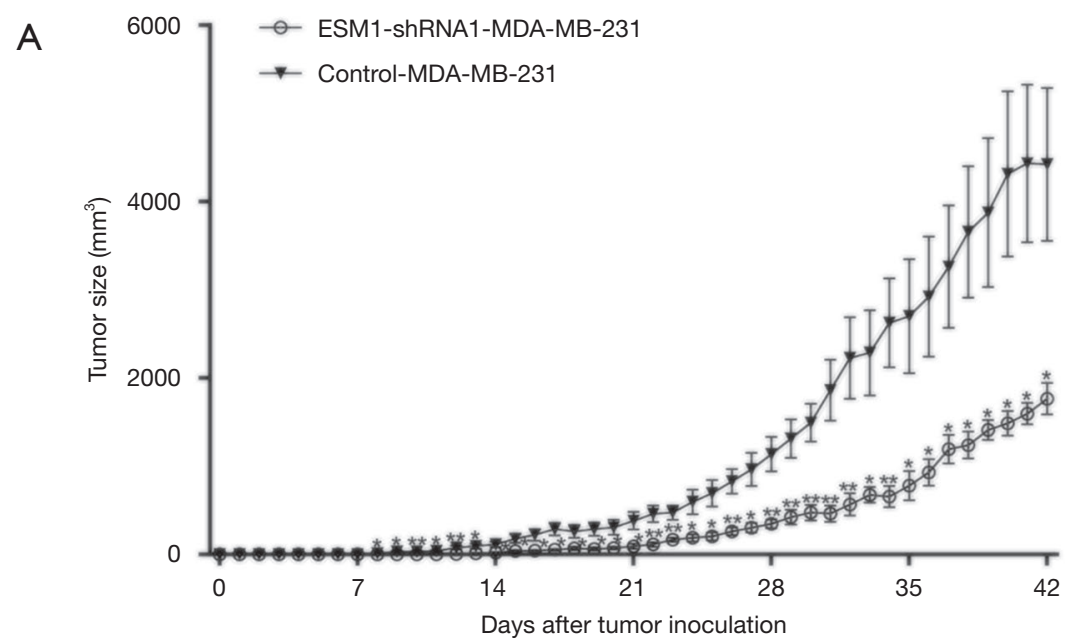

B Control-MDA-MB-231

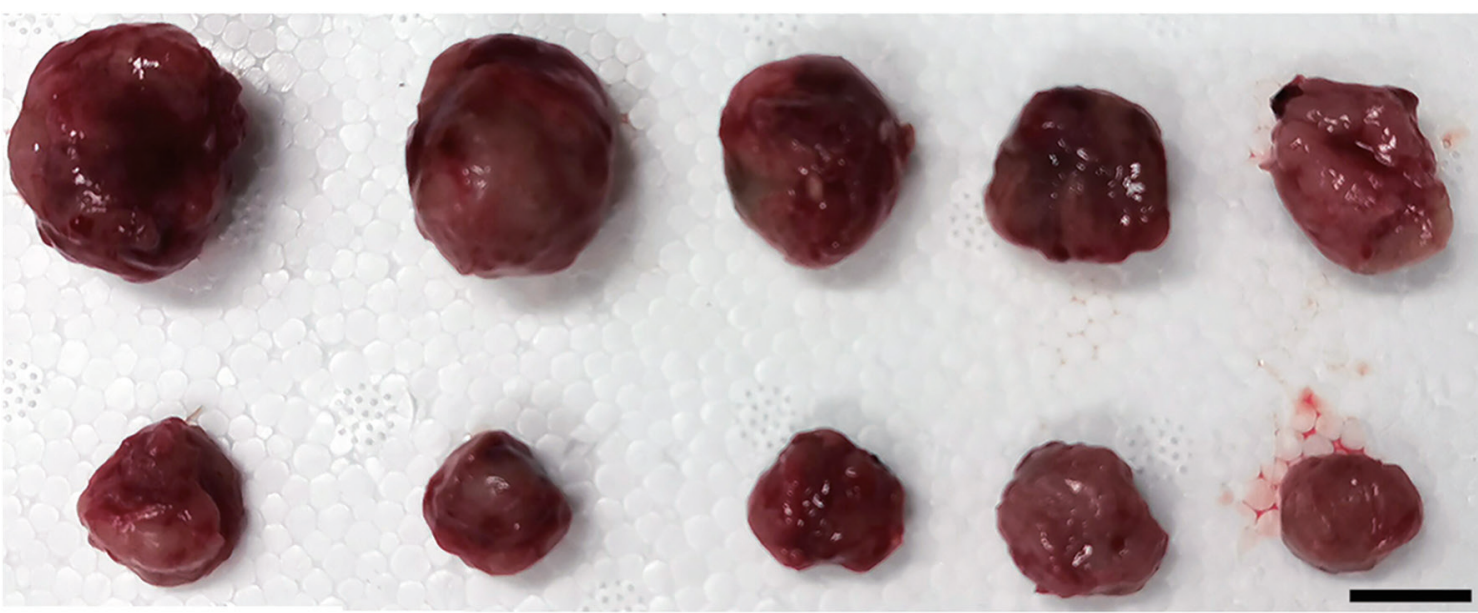

C ESM1-shRNA1-MDA-MB-231

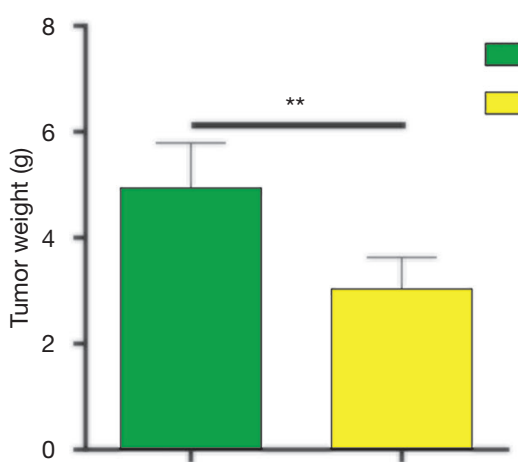

Control-MDA-MB-231

ESM1-shRNA1-MDA-MB-231

Figure 3 ESM1 knockdown inhibits in vivo progression of xenografted human TNBC in mice. (A) Tumor growth curve after subcutaneous inoculation of ESM1-shRNA1-MDA-MB-231 or Control-shRNA-MDA-MB-231 cells. (B) Images of dissected tumors. Scale bar, 10 mm. (C) Statistic analysis of tumor weight. $\mathrm{n}=5$ in each group. ${ }^{* *}, \mathrm{P}<0.01$. 
Page 10 of 18
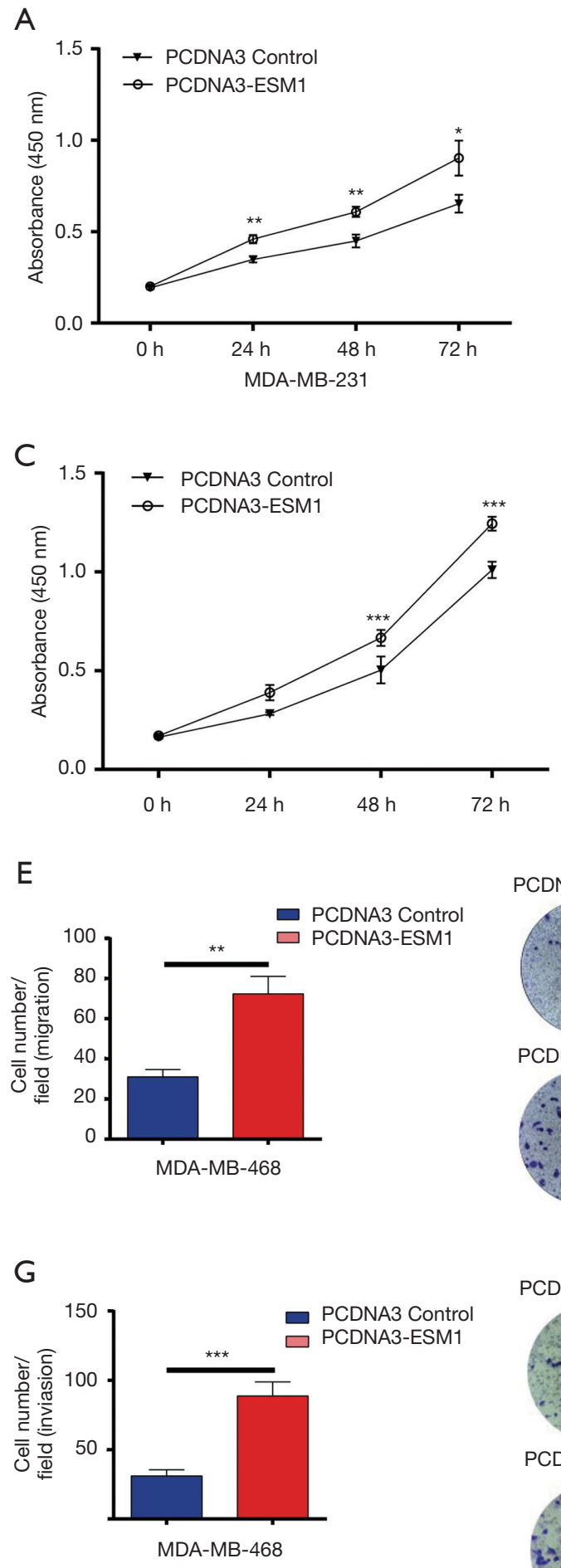

Liu et al. ESM1 promotes TNBC cell proliferation via AKT/NF-кB/Cyclin D
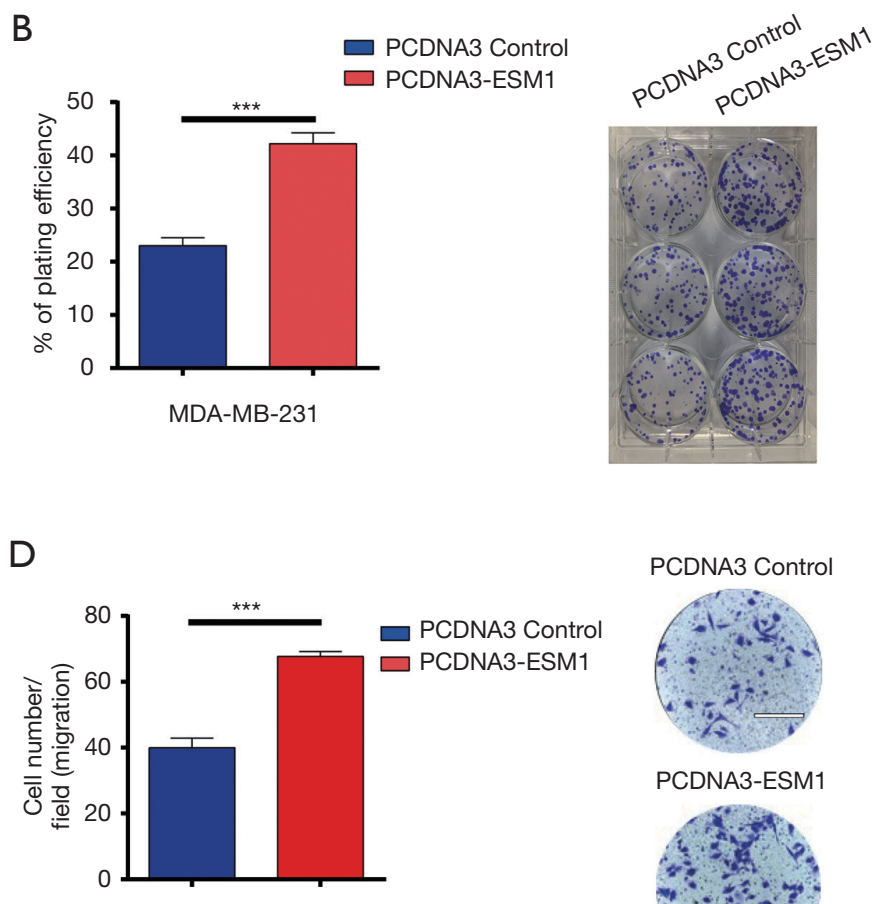

MDA-MB-231
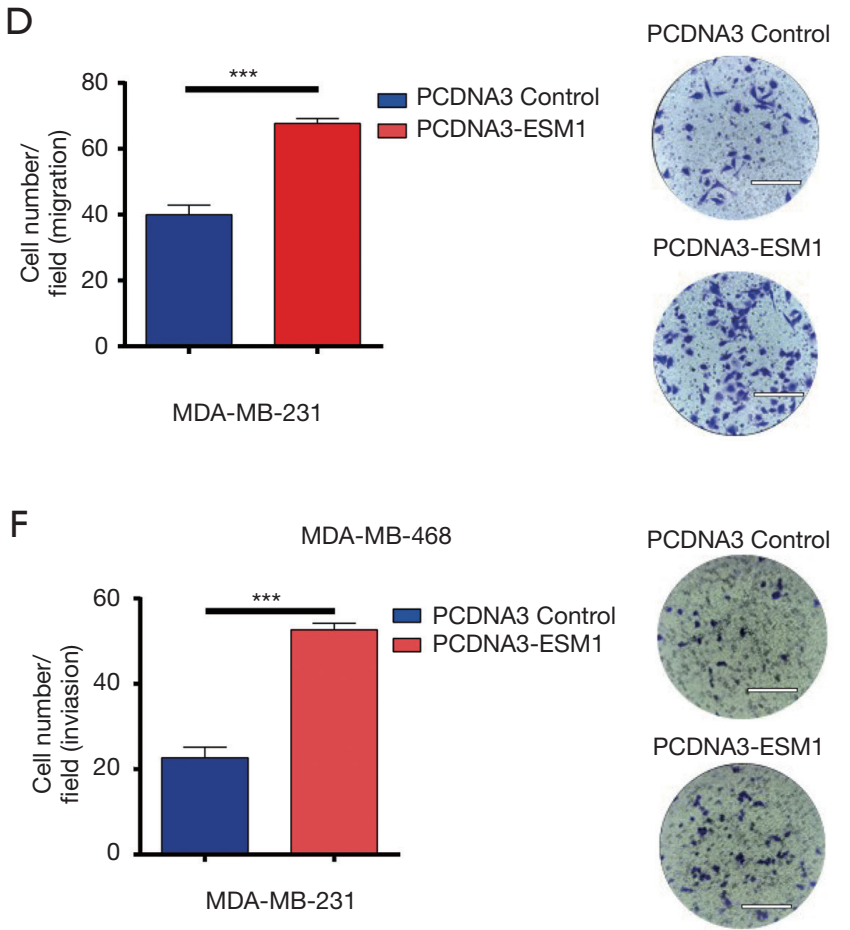

Figure 4 ESM1 overexpression enhances in vitro proliferation, migration and invasion of TNBC cell. (A) CCK-8 assay; (B) colony formation assay; (C) migration assay; (D) invasion assay on MDA-MB-231 cells transfected with ESM1-PCDNA3 and PCDNA3 control plasmids respectively; (E) CCK-8 assay; (F) migration assay; (G) invasion assay on MDA-MB-468 cells transfected with ESM1-PCDNA3 and PCDNA3 control plasmids respectively. Scale bar, $360 \mu \mathrm{m} .{ }^{*}, \mathrm{P}<0.05 ;{ }^{* *}, \mathrm{P}<0.01$; ***, $\mathrm{P}<0.001$. 
A

MDA-MB-23

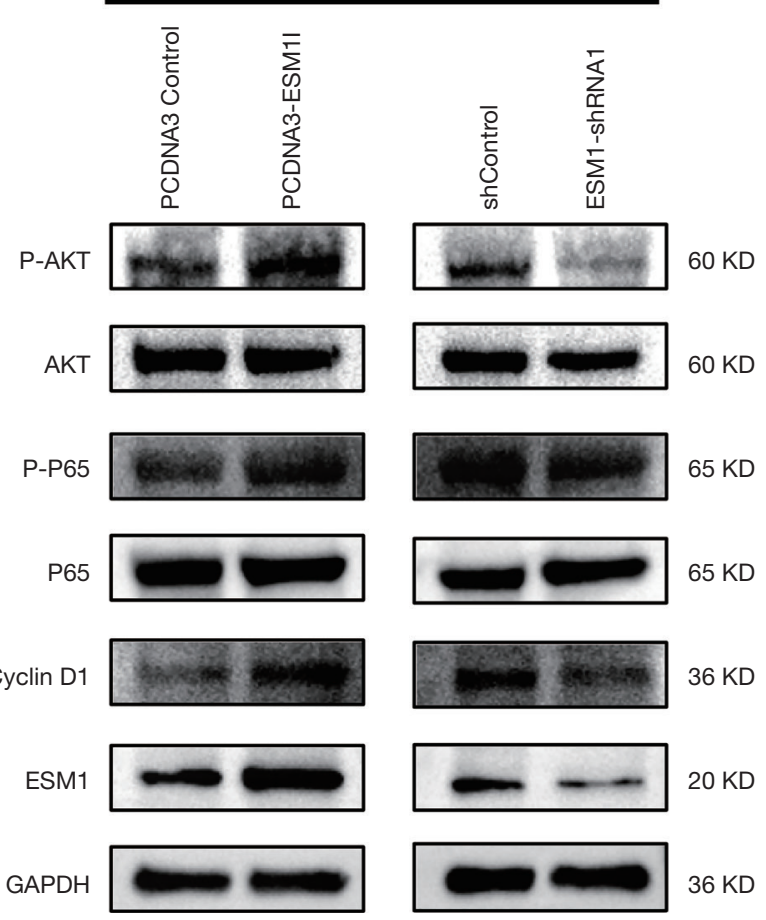

B

MDA-MB-468

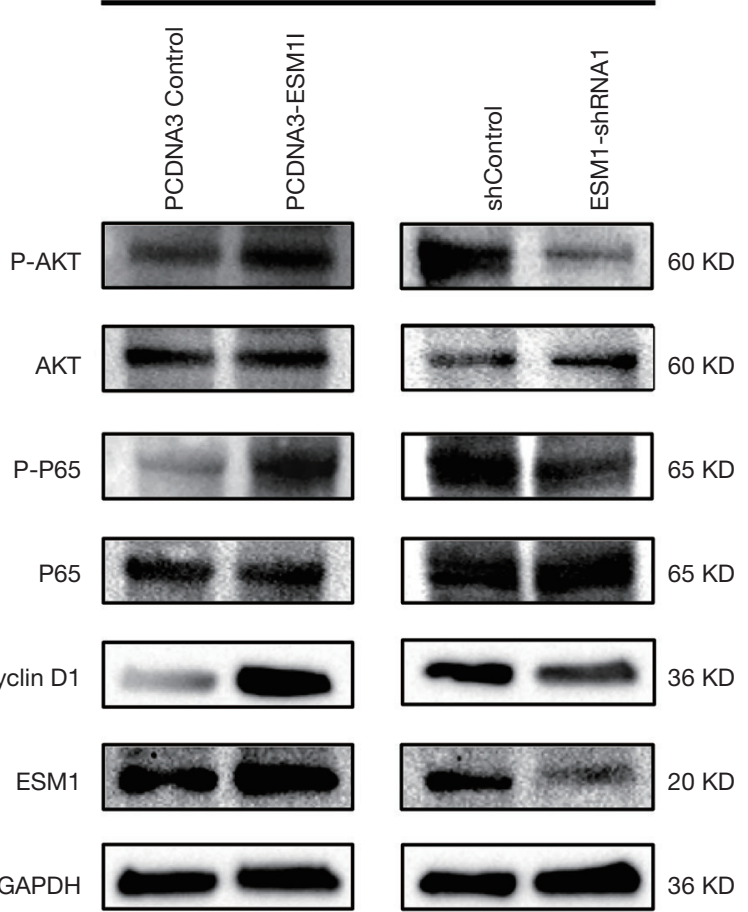

Figure 5 ESM1 activates the AKT/NF-kB/CyclinD1 signaling pathway. (A) Western blot analysis of Cyclin D1, P-AKT, P-p65, AKT, p65 and ESM1 in MDA-MB-231 cells when ESM1 was knocked down or overexpressed compared with the control group. (B) Western blot analysis of Cyclin D1, P-AKT, AKT, P-p65, p65 and ESM1 in MDA-MB-468 cells when ESM1 was knocked down or overexpressed compared with the control group. GAPDH was used as a loading control.

through activating an Akt-dependent NF- $\mathrm{B} / \mathrm{Cyclin}$ D1 pathway. The AKT inhibitor MK-2206 and NF$\kappa \mathrm{B}$ inhibitor BAY-117082 were then employed to further determine the function of AKT/NF- $\mathrm{B} / \mathrm{Cyclin} \mathrm{D} 1$ pathway in TNBC proliferation regulated by ESM1. The ESM1-overexpressing MDA-MB-231 and MDA-MB-468 cells were treated with MK-2206 and BAY-117082 at various concentrations for 1,2 and 3 days, and the cell proliferation was subsequently assessed by CCK- 8 assay. The results showed that treatment with MK-2206 or BAY117082 significantly inhibited the proliferation of ESM1overexpressing TNBC cell (Figure 6A,B,C,D). We further tested the expression of Cyclin D1 and the phosphorylation of AKT and NF- $\kappa \mathrm{B} / \mathrm{p} 65$ in ESM1-overexpressing TNBC cell after treatment with MK-2206 or BAY-117082 at $2 \mu \mathrm{M}$ or $5 \mu \mathrm{M}$ for 1 day. The results revealed that treatment of MK-2206 inhibits the phosphorylation of AKT and the expression of Cyclin D1 (Figure 6E) while treatment of BAY117082 inhibits the phosphorylation of NF- $\mathrm{kB} / \mathrm{p} 65$ and the expression of Cyclin D1 (Figure 6F). Together, our results indicate that ESM1 promotes TNBC cell proliferation by activating the AKT/NF- $\mathrm{kB} / \mathrm{CyclinD} 1$ pathway.

\section{Discussion}

TNBC does not respond to hormonal therapy medicines or treatments that target HER2. Probing the critical molecules that drive the progression of TNBC thus attracts tremendous research interest and is crucial to improve the treatment and prognosis of TNBC. ESM1 circulating in the bloodstream has been reported to increase with growing tumor volume (9). A higher ESM1 level has also been shown to be associated with high rate of metastasis and poor prognosis in several types of cancers $(21,22)$. Previous studies and our analyses using online resources of breast cancer patients revealed that ESM1 transcript level is significantly upregulated and the patients with higher ESM1 expression have worse prognosis. We therefore hypothesized that ESM1 plays an important role in TNBC progression. The hypothesis was confirmed 

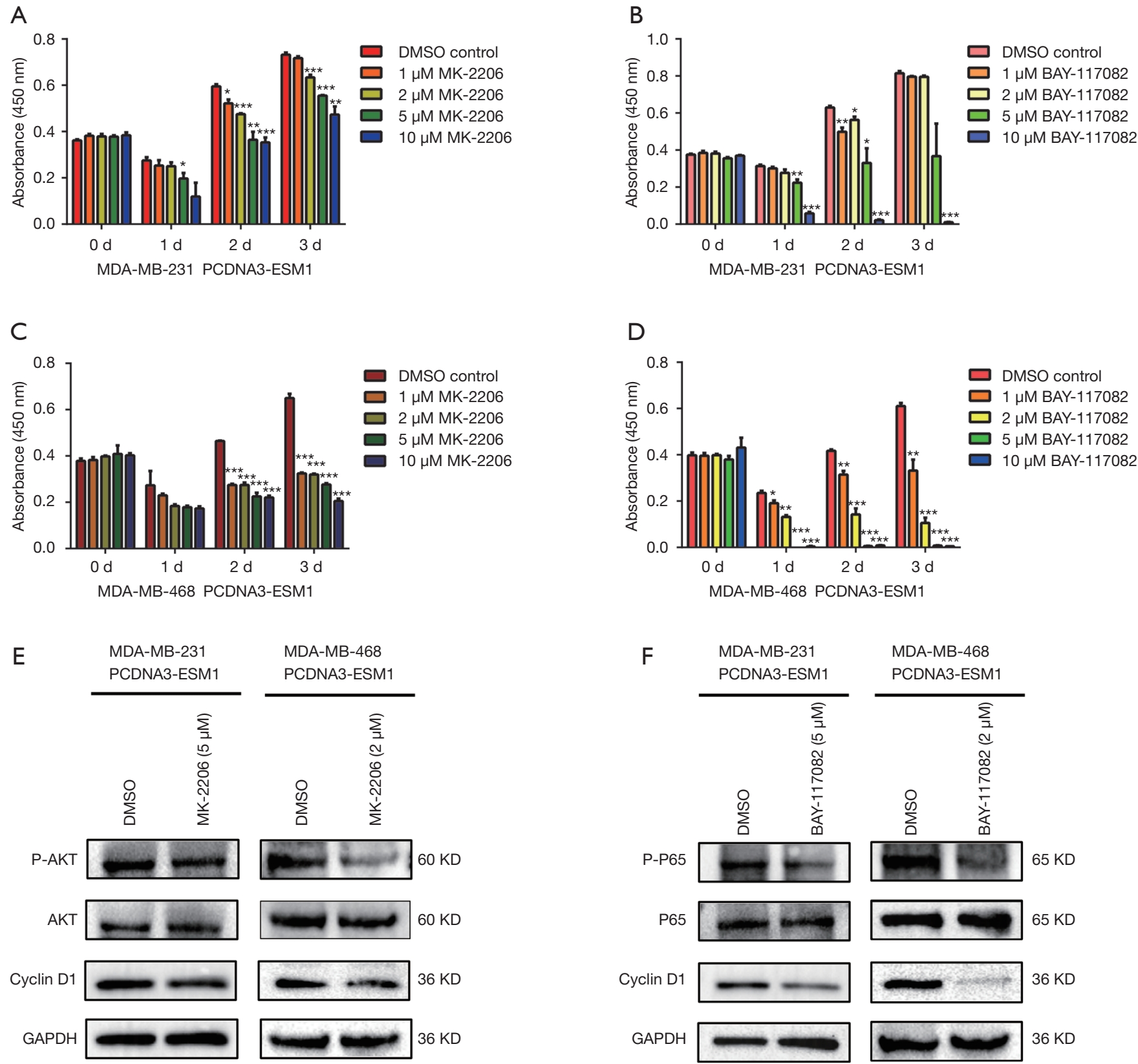

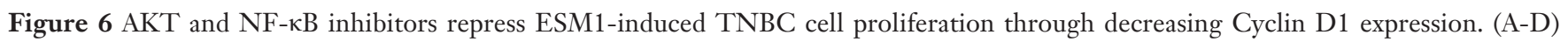
CCK-8 assay. MDA-MB-231 and MDA-MB-468 ESM1-overexpressing cells were treated with MK-2206 or BAY-117082 at various duration and concentrations; (E) Western blot result shows the expression of Cyclin D1, P-AKT, AKT in ESM1-overexpressing MDAMB-231 and MDA-MB-468 cells which were treated with MK-2206 at 2 or $5 \mu$ for 1 day. (F) Western blot result shows the expression of Cyclin D1, P-p65, p65 in ESM1-overexpressing MDA-MB-231 and MDA-MB-468 cells which were treated with BAY-117082 at 2 or $5 \mu$ M for 1 day. *, $\mathrm{P}<0.05 ;$ **, $\mathrm{P}<0.01$; *** $\mathrm{P}<0.001$. 
Table 2 Drugs targeting the upstream and downstream molecules of ESM1 for the treatment of TNBC

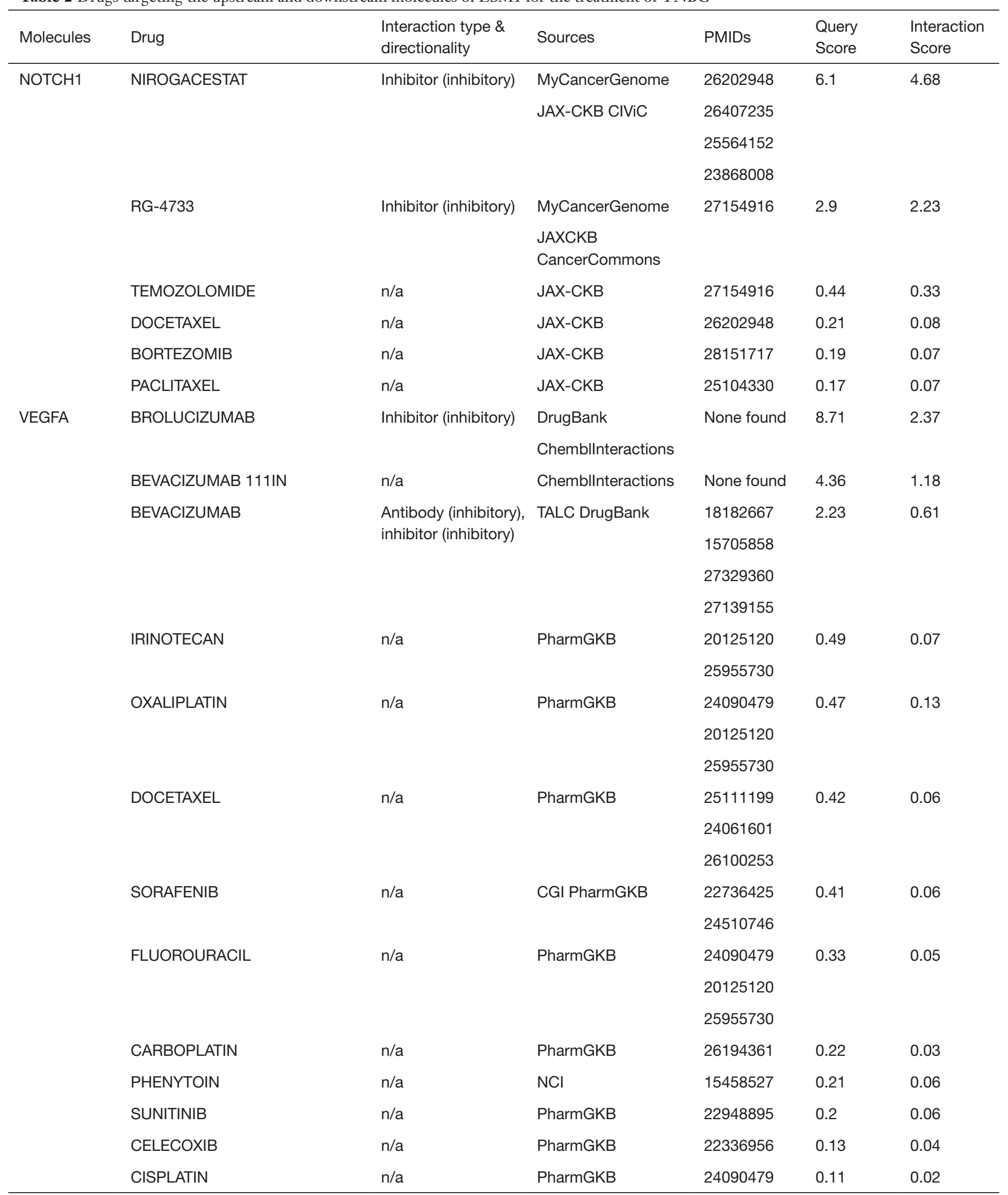

Table 2 (continued) 
Table 2 (continued)

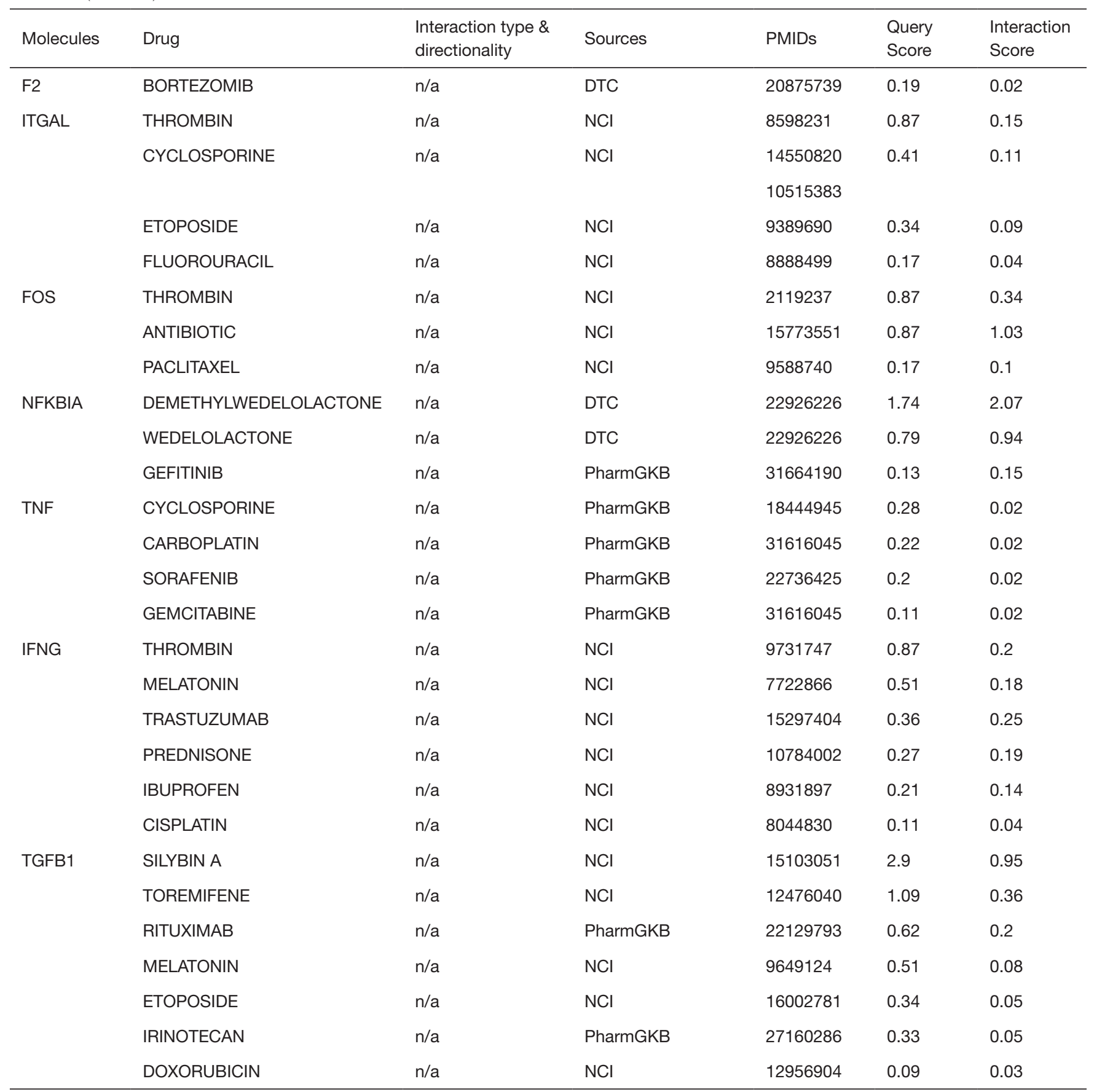

n/a, not available.

and the underlying cellular mechanism was unraveled by the following findings of this study: ESM1 knockdown decreases in vitro proliferation, migration and invasion of TNBC cells and inhibits in vivo tumor growth in xenograft mouse model; ESM1 overexpression increases in vitro
TNBC cell proliferation, migration and invasion.

In the process of clinical diagnosis, it is often necessary to take biopsy from the tumor site for examination. Since ESM1 expression was significantly up-regulated in breast cancer tissues with different stages, lymph node metastasis 
and subtypes, it potentially can be used as a biomarker for early diagnosis of breast cancer. However, repeated sampling from the tumor or sampling at the early stage is difficult, while liquid biopsy from the blood is more convenient and allows repeated sampling. ESM1 is a secreted protein which is mainly expressed in the endothelial cells including the vascular endothelium, and has been found freely circulating in the bloodstream of healthy tissues. Therefore, ESM1 has advantages compared with other non-blood-derived molecules to serve as a diagnostic or prognostic indicator.

The ESM1 in the circulation or the tumor microenvironment might also play an important role in modulating the progression of breast cancer, which is not examined in this study but remains an interesting direction for further investigation. On the other hand, the expression of ESM1 has been shown to be concomitant with that of angiogenic markers (23), and ESM1 can enhance the tumor angiogenesis through interacting with vascular endothelial growth factor (VEGF) (10). Taken together with our findings, it is very possible that ESM1 can promote the progression of TNBC at multiple levels.

Our study has disclosed that ESM1 plays an important role the progression of TNBC, while the molecular mechanism remains elusive. Cyclin D1 is a key regulatory factor of the G1 to $\mathrm{S}$ transition during cell cycle and promotes cell cycle progression by phosphorylating and inactivating the retinoblastoma protein (RB) (24). The expression of Cyclin D1 is regulated by various signaling pathways including AKT/NF-кB signaling pathway (16). AKT can be activated by growth factors or DNA damage to regulate the proliferation, survival and metabolism of tumor cells $(25,26)$. NF- $\kappa \mathrm{B}$ is a key transcription factor that links inflammation with cancer and serves as a key player in the immune system, cell survival, proliferation and apoptosis $(27,28)$. Misregulation or constitutive activation of the NF$\kappa \mathrm{B}$ pathway is involved in tumorigenesis of breast cancer and is associated with the hormone-independent tumor growth (29). In this study, we found that ESM1 expression was positively associated with the activity of AKT/NF- $\mathrm{BB} /$ Cyclin D1 pathway. The expression of Cyclin D1 as well as phosphorylation of $\mathrm{AKT}$ and $\mathrm{NF}-\kappa \mathrm{B}$ in both MDAMB-231 and MDA-MB-468 cells were upregulated when ESM1 was overexpressed while downregulated when ESM1 was knocked down. We thus presumed that ESM1 promotes TNBC cell proliferation through activating AKT/NF- $\mathrm{kB} /$ Cyclin D1 pathway. The hypothesis was validated by the fact that the elevated phosphorylation of AKT and NF- $\mathrm{KB}$ as well as increased Cyclin D1 expression was prevented by treatment of AKT inhibitor MK-2206 and NF- $\mathrm{KB}$ inhibitor BAY-117082 respectively and that the ESM1-induced overproliferation is accordingly suppressed. Therefore, targeting ESM1 is potentially an effective strategy to treat TNBC as it can inhibit the proliferation of tumor cells and alter the activity of AKT/NF-кB/Cyclin D1 pathway. It remains elusive in terms of how ESM1 modulates AKT/ $\mathrm{NF}-\kappa \mathrm{B}$ activity, the disclosure of which will help further understanding the molecular mechanisms that dominate the proliferation of TNBC cell.

Other molecules and pathways, besides AKT/NF$\kappa \mathrm{B} / \mathrm{Cyclin} \mathrm{D} 1$ pathway, might be also involved in ESM1 promoting TNBC progression. It has been shown that ESM1 expression can be stimulated by VEGFA through the phosphorylation and activation of VEGFR-2, which is required to promote the angiogenesis in bladder cancer (10). Additionally, ESM1 has been classified as a member of the insulin-like growth factor binding protein (IGFBP) superfamily and is associated with insulin-like growth factor (IGF) to promote cell growth and proliferation $(5,30)$. Previous studies have also shown that ESM1 expression can be induced by TNF $\alpha$ or IL-1b and downregulated by IFN $\gamma$ (31). We further explored the regulation network of ESM1 using IPA (www.qiagen.com/ingenuity) tools (Figure S3). VEGFA and TNF, which have been shown to regulate ESM1 expression, are positive hits in the predicted regulation network. Besides VEGFA and TNF, we also found other candidate molecules that might be important players in the upstream regulatory network of ESM1, e.g., FOS and PPRC1, the alterations of which have been demonstrated to be associate with TNBC $(32,33)$. On the other hand, intercellular adhesion molecule 1 (ICAM1), which is highly expressed in TNBC tissue and cell lines (34), is the predicted downstream gene and might be regulated by ESM1.

By searching the present drugs used in the treatment of breast cancer, we did not find any drug that is reported to directly inhibit the expression of ESM1. However, previous studies have reported that ESM1 is regulated by VEGFA, PI3K and MAPK $(35,36)$. Therefore, drugs that can alter the activity of VEGFA, PI3K and MAPK are supposed to regulate ESM1 expression. In order to explore the drugs targeting the upstream and downstream molecules of esm1 for the treatment of TNBC, we took advantage of the online resource of Drug-Gene Interaction database (https://dgidb.genome.wustl.edu/). There are many drugs targeting the upstream and downstream molecules of ESM1 (Table 2 in total online: https://cdn.amegroups.cn/static/ 
public/atm-20-7005-5.xlsx). Among them, some drugs such as Bevacizumab, Carboplatin, Cisplatin, Doxorubicin, Gefitinib, Paclitaxel, and Temozolomide have been used in clinical treatment of TNBC.

Besides ESM1, many other molecules have been shown to be highly expressed in TNBC tissues and drive the progress of TNBC through various mechanisms. For example, $\mathrm{XBP} 1$ plays a pivotal role in the tumorigenicity and progression of TNBC (37). Phosphatase PTP4A3 promotes TNBC growth and high expression of PTP4A3 could serve as a prognostic indicator for worse overall survival (38). In addition, TRA2A, ADAM-17, Cx26, BCL11A, Annexin A1, and Jagged2 have also been proved to drive the progress of TNBC (39-44).

\section{Acknowledgments}

Funding: This work is supported by National Natural Science Foundation of China (Grant No. 31871481 , 32070973, 31850410471, 31571107), Natural Science Foundation of Hubei Province (Grant No. 2017CFA064), Fundamental Research Funds for the Central Universities (Program No. 2662017PY109, 2662018PY026, 2015RC011).

\section{Footnote}

Reporting Checklist: The authors have completed the ARRIVE reporting checklist. Available at http://dx.doi. org/10.21037/atm-20-7005org/10.21037/atm-20-7005

Data Sharing Statement: Available at http://dx.doi. org/10.21037/atm-20-7005

Conflicts of Interest: All authors have completed the ICMJE uniform disclosure form (available at http://dx.doi. org/10.21037/atm-20-7005). The authors have no conflicts of interest to declare.

Ethical Statement: The authors are responsible for all aspects of the work to ensure that issues related to the accuracy or integrity of any part of the work are properly investigated and resolved. All animal experiments were approved by the Scientific Ethics Committee of Huazhong Agricultural University (permit number HZAUMO-2015-016). Experiments were performed in compliance with Chinese national or institutional guidelines for the care and use of animals.
Open Access Statement: This is an Open Access article distributed in accordance with the Creative Commons Attribution-NonCommercial-NoDerivs 4.0 International License (CC BY-NC-ND 4.0), which permits the noncommercial replication and distribution of the article with the strict proviso that no changes or edits are made and the original work is properly cited (including links to both the formal publication through the relevant DOI and the license). See: https://creativecommons.org/licenses/by-nc-nd/4.0/.

\section{References}

1. Alimirzaie S, Bagherzadeh M, Akbari MR. Liquid biopsy in breast cancer: A comprehensive review. Clin Genet 2019;95:643-60.

2. M Braden A, V Stankowski R, M Engel J, et al. Breast cancer biomarkers: risk assessment, diagnosis, prognosis, prediction of treatment efficacy and toxicity, and recurrence. Curr Pharm Des 2014;20:4879-98.

3. Mendez O, Perez J, Soberino J, et al. Clinical Implications of Extracellular HMGA1 in Breast Cancer. Int J Mol Sci 2019;20:5950.

4. Ishikawa $\mathrm{Y}$, Horiguchi J, Toya H, et al. Triplenegative breast cancer: histological subtypes and immunohistochemical and clinicopathological features. Cancer Sci 2011;102:656-62.

5. Sarrazin S, Adam E, Lyon M, et al. Endocan or endothelial cell specific molecule-1 (ESM-1): a potential novel endothelial cell marker and a new target for cancer therapy. Biochim Biophys Acta 2006;1765:25-37.

6. Kang YH, Ji NY, Han SR, et al. ESM-1 regulates cell growth and metastatic process through activation of NFkappa B in colorectal cancer. Cell Signal 2012;24:1940-9.

7. Grigoriu BD, Depontieu F, Scherpereel A, et al. Endocan expression and relationship with survival in human nonsmall cell lung cancer. Clin Cancer Res 2006;12:4575-82.

8. El Behery MM, Seksaka MA, Ibrahiem MA, et al. Clinicopathological correlation of endocan expression and survival in epithelial ovarian cancer. Arch Gynecol Obstet 2013;288:1371-6.

9. Scherpereel A, Gentina T, Grigoriu B, et al. Overexpression of endocan induces tumor formation. Cancer Res 2003;63:6084-9.

10. Roudnicky F, Poyet C, Wild P, et al. Endocan is upregulated on tumor vessels in invasive bladder cancer where it mediates VEGF-A-induced angiogenesis. Cancer Res 2013;73:1097-106.

11. Suraj J, Kurpinska A, Zakrzewska A, et al. Early and late 
endothelial response in breast cancer metastasis in mice: simultaneous quantification of endothelial biomarkers using a mass spectrometry-based method. Dis Model Mech 2019;12:dmm036269.

12. Sagara A, Igarashi K, Otsuka M, et al. Endocan as a prognostic biomarker of triple-negative breast cancer. Breast Cancer Res Treat 2017;161:269-78.

13. Burnett RM, Craven KE, Krishnamurthy P, et al. Organspecific adaptive signaling pathway activation in metastatic breast cancer cells. Oncotarget 2015;6:12682-96.

14. Jin H, Rugira T, Ko YS, et al. ESM-1 Overexpression is Involved in Increased Tumorigenesis of RadiotherapyResistant Breast Cancer Cells. Cancers 2020;12:1363.

15. Engelman JA, Luo J, Cantley LC. The evolution of phosphatidylinositol 3-kinases as regulators of growth and metabolism. Nat Rev Genet 2006;7:606-19.

16. Qie S, Diehl JA. Cyclin D1, cancer progression, and opportunities in cancer treatment. J Mol Med (Berl) 2016;94:1313-26.

17. Wu Y, Tang W, Cao Y, et al. A Cyclin D1-Specific Single-Chain Variable Fragment Antibody that Inhibits HepG2 Cell Growth and Proliferation. Biotechnol J 2020;15:e1900430.

18. Ruland J. Return to homeostasis: downregulation of NFkappa B responses. Nat Immunol 2011;12:709-14.

19. Fornier MN, Rathkopf D, Shah M, et al. Phase I dosefinding study of weekly docetaxel followed by flavopiridol for patients with advanced solid tumors. Clin Cancer Res 2007;13:5841-6.

20. Liu W'T, Lv YJ, Yang RC, et al. New insights into meningitic Escherichia coli infection of brain microvascular endothelial cells from quantitative proteomics analysis. J Neuroinflammation 2018;15:291.

21. Bhalla S, Kaur H, Dhall A, et al. Prediction and Analysis of Skin Cancer Progression using Genomics Profiles of Patients. Sci Rep 2019;9:15790.

22. Cai H, Yang X, Gao Y, et al. Exosomal MicroRNA-9-3p Secreted from BMSCs Downregulates ESM1 to Suppress the Development of Bladder Cancer. Mol Ther Nucleic Acids 2019;18:787-800.

23. Gerritsen ME, Tomlinson JE, Zlot C, et al. Using gene expression profiling to identify the molecular basis of the synergistic actions of hepatocyte growth factor and vascular endothelial growth factor in human endothelial cells. Br J Pharmacol 2003;140:595-610.

24. Giacinti C, Giordano A. RB and cell cycle progression. Oncogene 2006;25:5220-7.

25. Manning BD, Cantley LC. AKT/PKB signaling: navigating downstream. Cell 2007;129:1261-74.

26. Basho RK, Gilcrease M, Murthy RK, et al. Targeting the PI3K/AKT/mTOR Pathway for the Treatment of Mesenchymal Triple-Negative Breast Cancer: Evidence From a Phase 1 Trial of mTOR Inhibition in Combination With Liposomal Doxorubicin and Bevacizumab. JAMA Oncol 2017;3:509-15.

27. Zhang Q, Lenardo MJ, Baltimore D. 30 Years of NF-kappaB: A Blossoming of Relevance to Human Pathobiology. Cell 2017;168:37-57.

28. Khongthong P, Roseweir AK, Edwards J. The NF-KB pathway and endocrine therapy resistance in breast cancer. Endocr Relat Cancer 2019;26:R369-80.

29. Zhou Y, Eppenberger-Castori S, Marx C, et al. Activation of nuclear factor-kappaB (NFkappaB) identifies a highrisk subset of hormone-dependent breast cancers. Int J Biochem Cell Biol 2005;37:1130-44.

30. Ma Y, Lu B, Ruan W, et al. Tumor suppressor gene insulin-like growth factor binding protein-related protein 1 (IGFBP-rP1) induces senescence-like growth arrest in colorectal cancer cells. Exp Mol Pathol 2008;85:141-5.

31. Lassalle P, Molet S, Janin A, et al. ESM-1 is a novel human endothelial cell-specific molecule expressed in lung and regulated by cytokines. J Biol Chem 1996;271:20458-64.

32. Serino LTR, Jucoski TS, Morais SB, et al. Association of FOSL1 copy number alteration and triple negative breast tumors. Genet Mol Biol 2019;42:26-31.

33. Willis S, De P, Dey N, et al. Enriched transcription factor signatures in triple negative breast cancer indicates possible targeted therapies with existing drugs. Meta Gene 2015;4:129-41.

34. Guo P, Huang J, Wang L, et al. ICAM-1 as a molecular target for triple negative breast cancer. Proc Natl Acad Sci U S A 2014;111:14710-5.

35. Rennel E, Mellberg S, Dimberg A, et al. Endocan is a VEGF-A and PI3K regulated gene with increased expression in human renal cancer. Exp Cell Res 2007;313:1285-94.

36. Zuo L, Lu M, Zhou Q, et al. Butyrate suppresses proliferation and migration of RKO colon cancer cells though regulating endocan expression by MAPK signaling pathway. Food Chem Toxicol 2013;62:892-900.

37. Chen X, Iliopoulos D, Zhang Q, et al. XBP1 promotes triple-negative breast cancer by controlling the HIF1alpha pathway. Nature 2014;508:103-7.

38. den Hollander P, Rawls K, Tsimelzon A, et al. Phosphatase PTP4A3 Promotes Triple-Negative Breast Cancer Growth and Predicts Poor Patient Survival. Cancer Res 
2016;76:1942-53.

39. Khaled WT, Choon Lee S, Stingl J, et al. BCL11A is a triple-negative breast cancer gene with critical functions in stem and progenitor cells. Nat Commun 2015;6:5987.

40. Li CY, Miao KL, Chen Y, et al. Jagged2 promotes cancer stem cell properties of triple negative breast cancer cells and paclitaxel resistance via regulating microRNA-200. Eur Rev Med Pharmacol Sci 2018;22:6008-14.

41. Liu T, Sun H, Zhu D, et al. TRA2A Promoted Paclitaxel Resistance and Tumor Progression in Triple-Negative Breast Cancers via Regulating Alternative Splicing. Mol

Cite this article as: Liu W, Yang Y, He B, Ma F, Sun F, Guo M, Zhang M, Dong Z. ESM1 promotes triple-negative breast cancer cell proliferation through activating AKT/NF- $\mathrm{\kappa B} / \mathrm{Cyclin}$ D1 pathway. Ann Transl Med 2021;9(7):533. doi: 10.21037/ atm-20-7005
Cancer Ther 2017;16:1377-88.

42. McGowan PM, Mullooly M, Caiazza F, et al. ADAM-17: a novel therapeutic target for triple negative breast cancer. Ann Oncol 2013;24:362-9.

43. Okano M, Kumamoto K, Saito M, et al. Upregulated Annexin A1 promotes cellular invasion in triple-negative breast cancer. Oncol Rep 2015;33:1064-70.

44. Thiagarajan PS, Sinyuk M, Turaga SM, et al. Cx26 drives self-renewal in triple-negative breast cancer via interaction with NANOG and focal adhesion kinase. Nat Commun 2018;9:578. 


\section{FIGURE 1D}
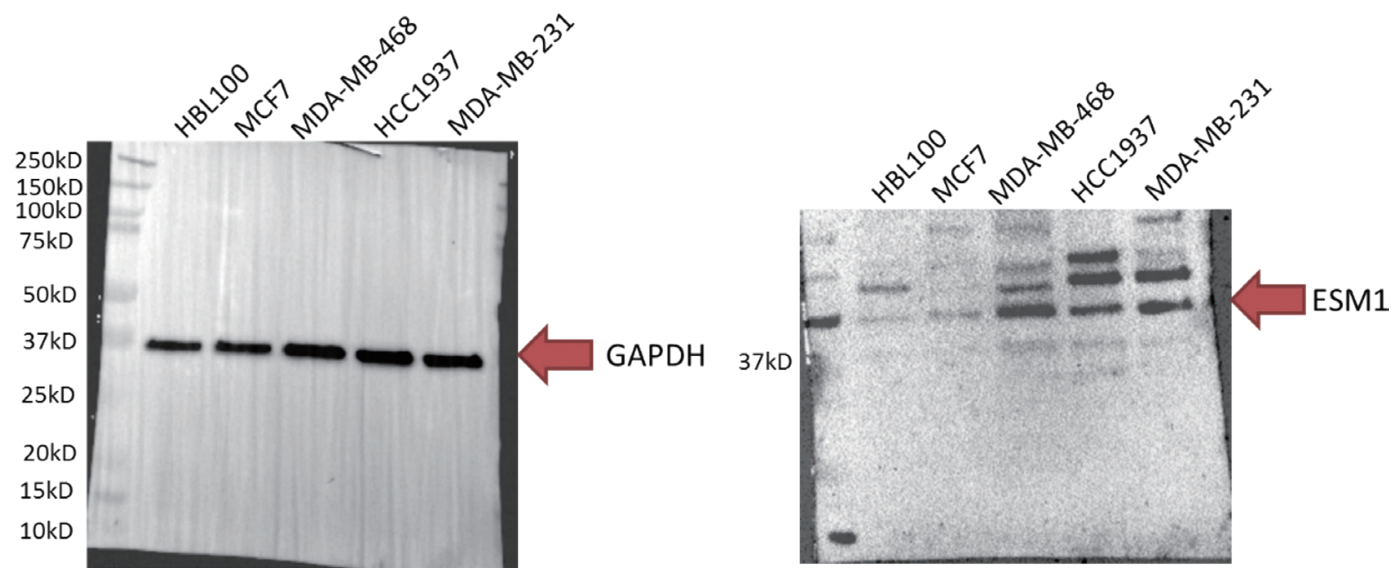

FIGURE 5A
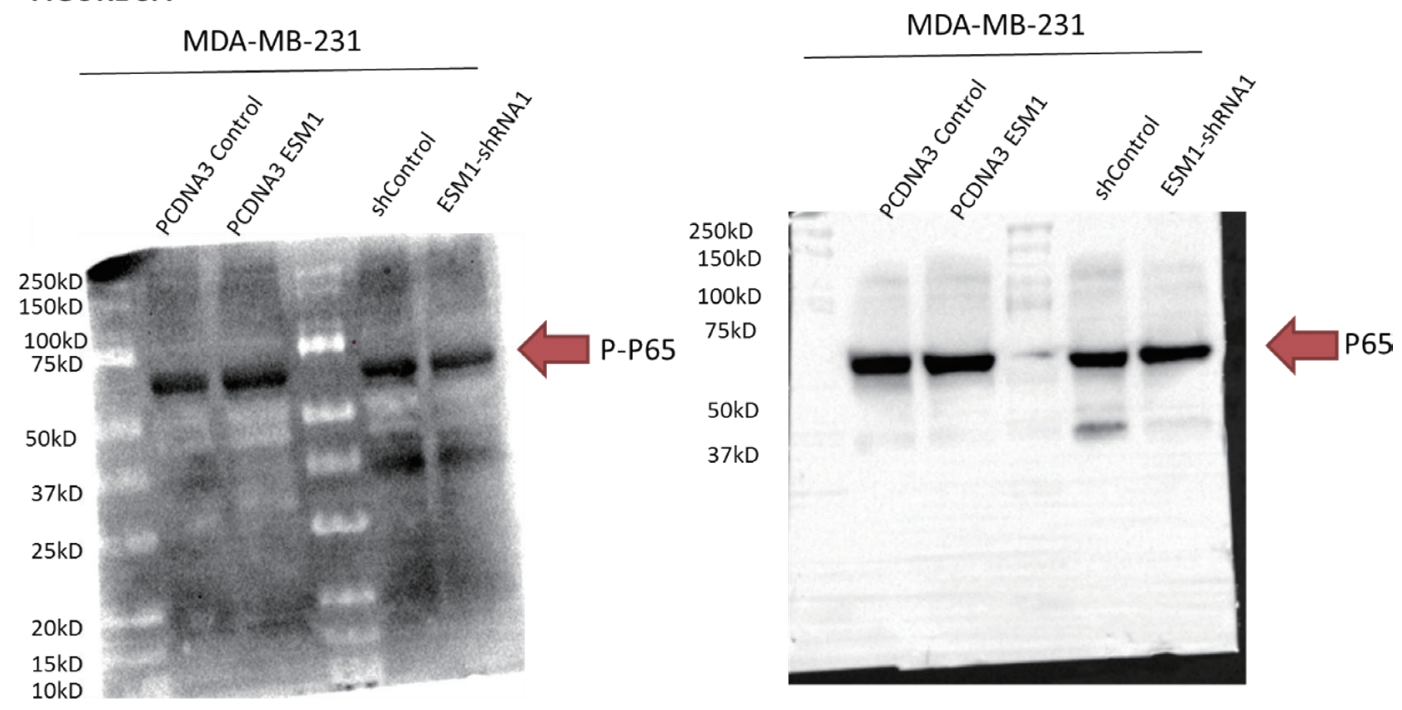

FIGURE 5A
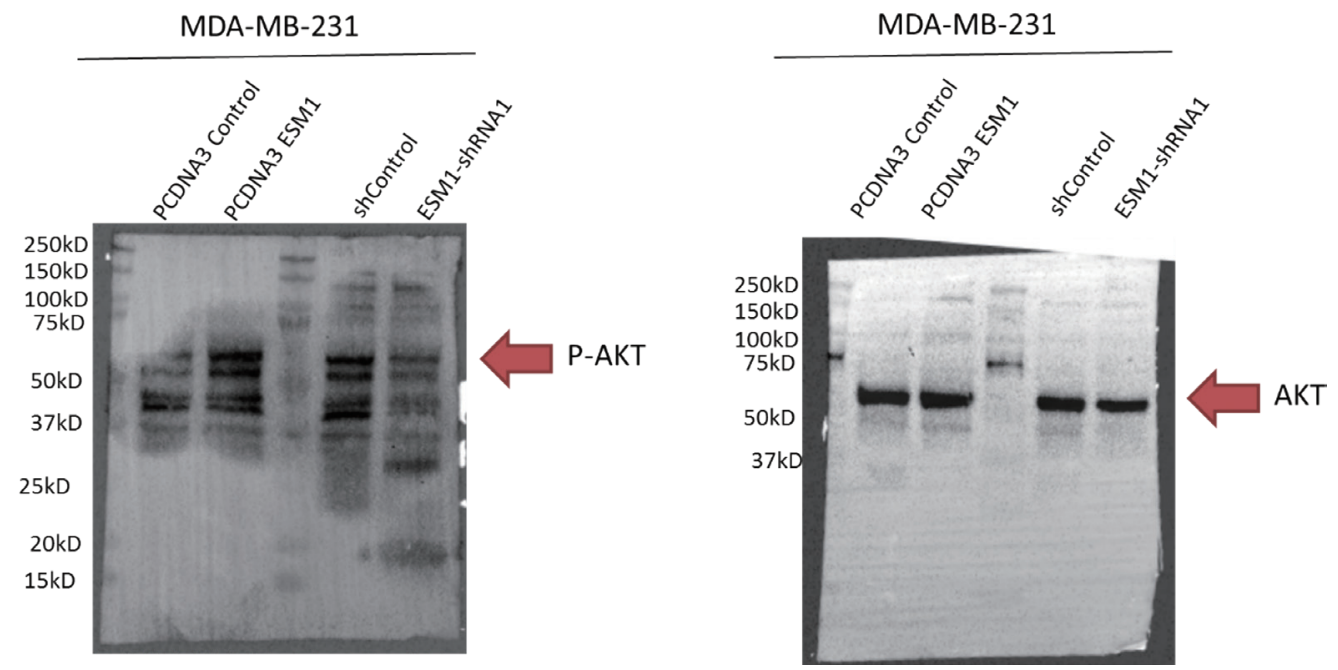

(c) Annals of Translational Medicine. All rights reserved. 
FIGURE 5A

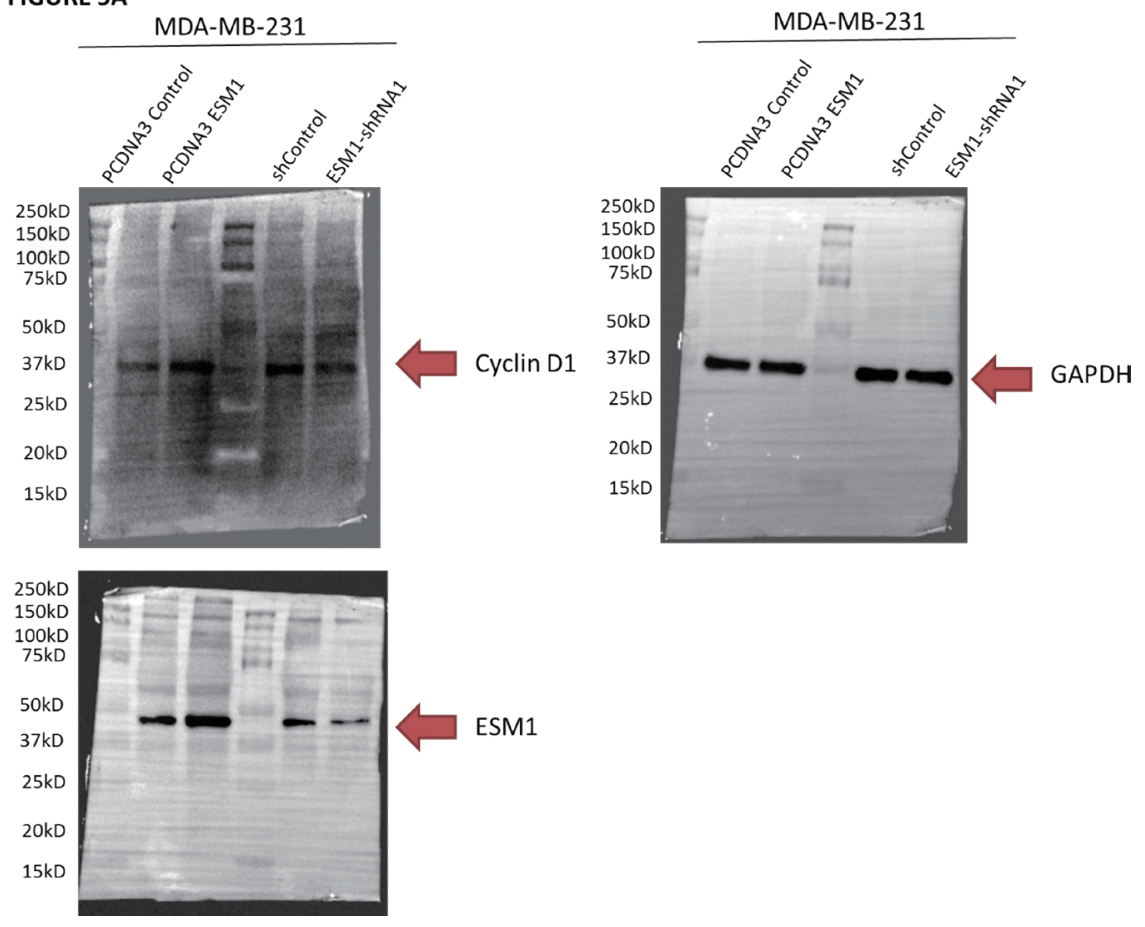

FIGURE 5B

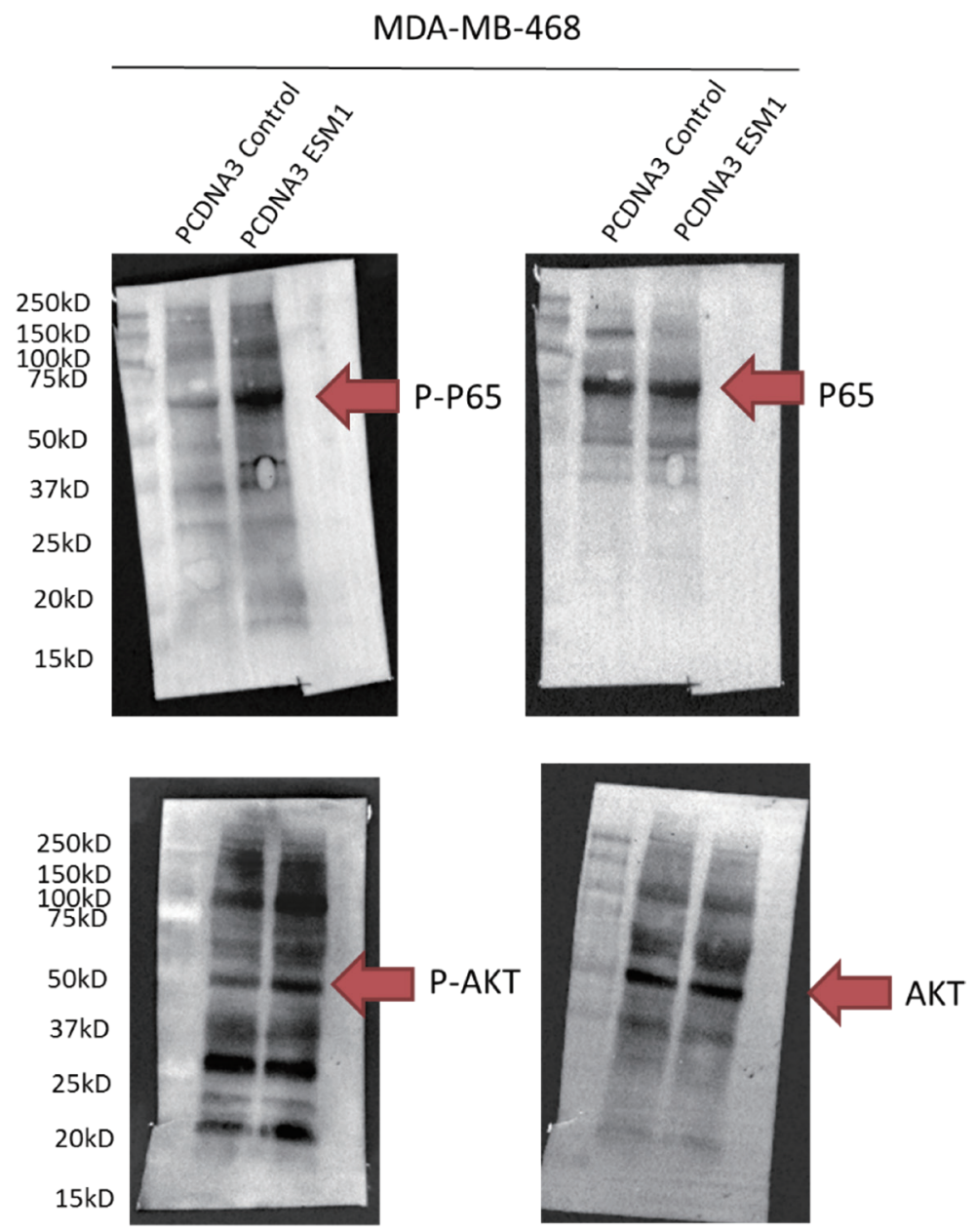




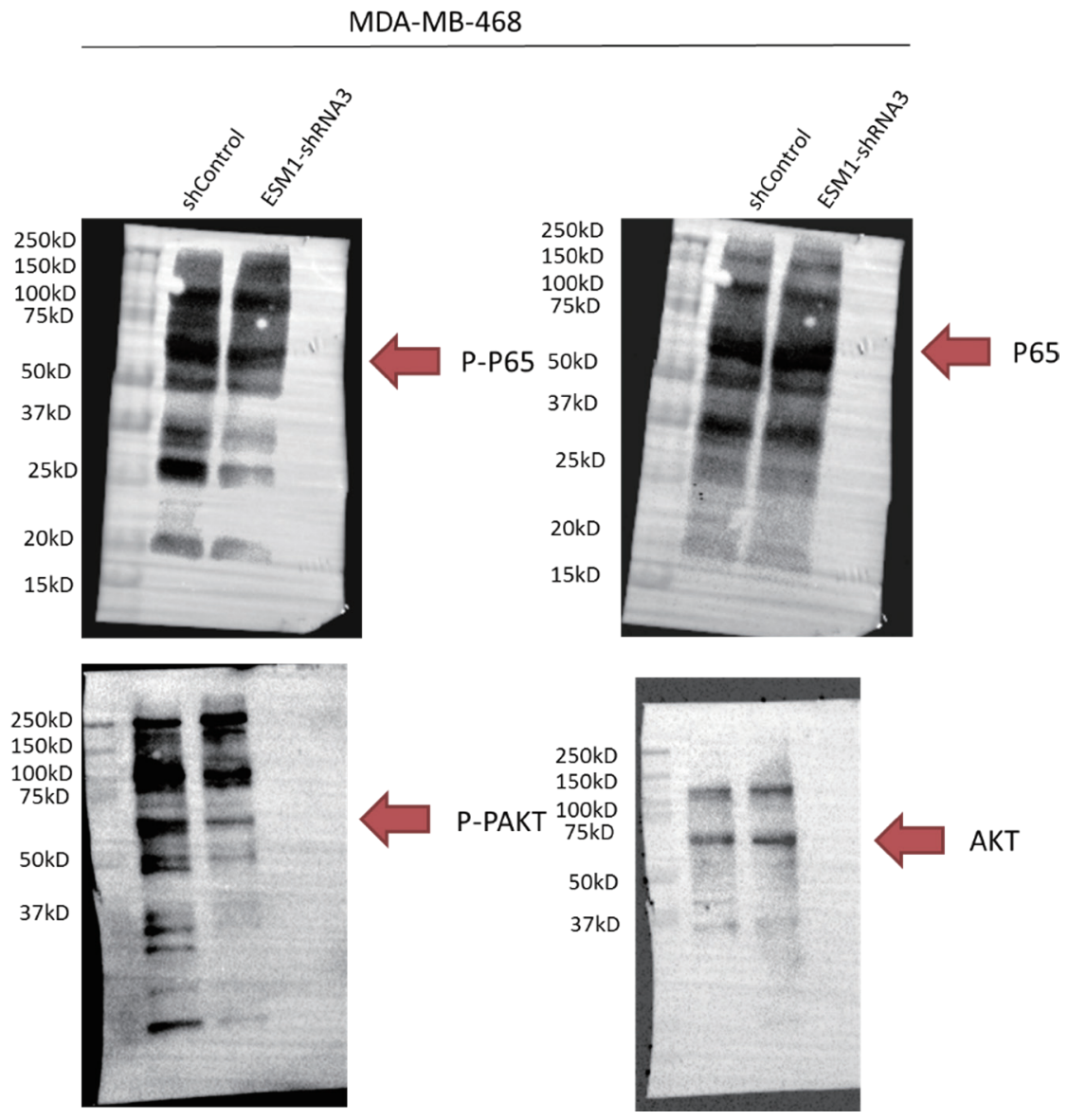

FIGURE 5B

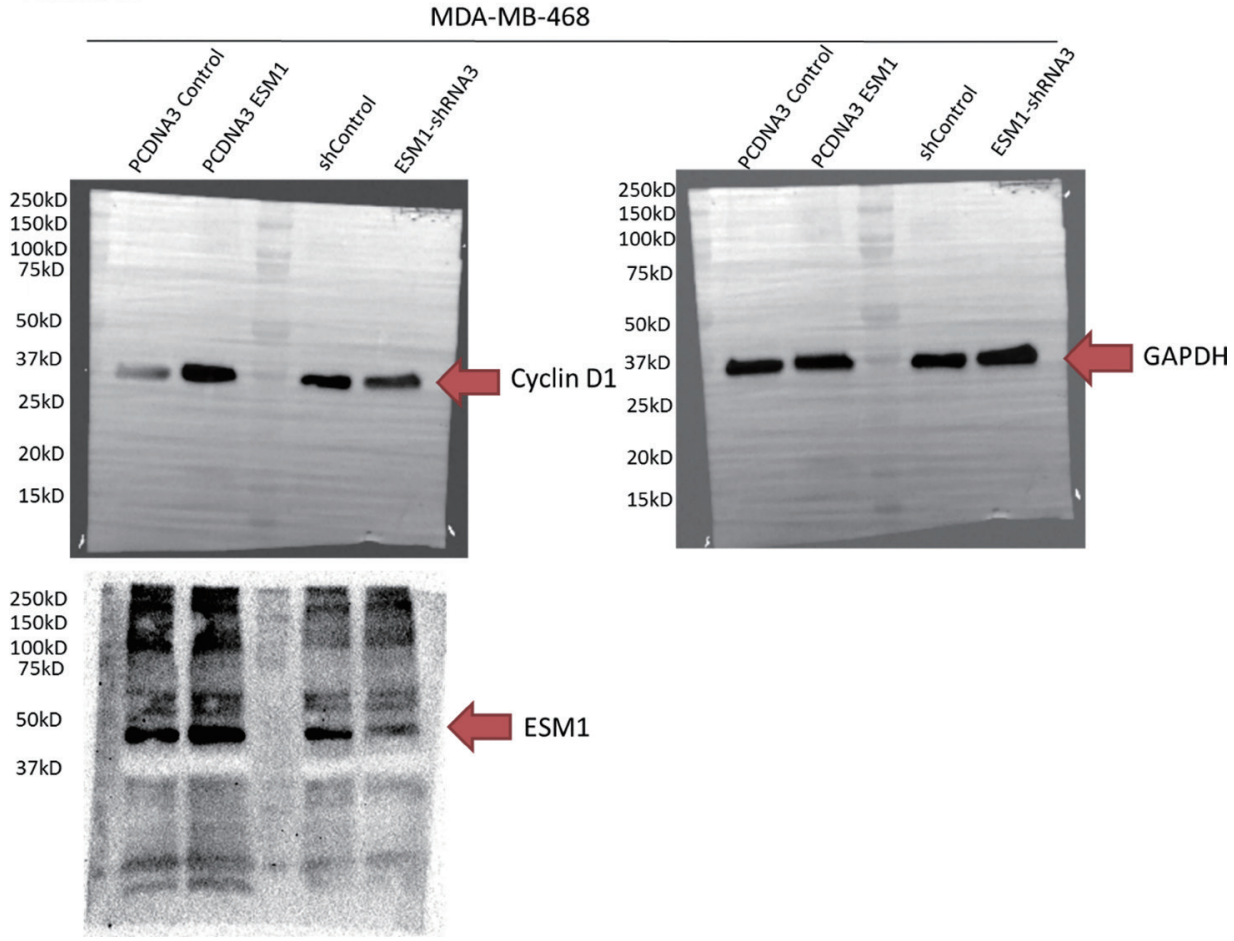


FIGURE 6E

MDA-MB-231 PCDNA3-ESM1
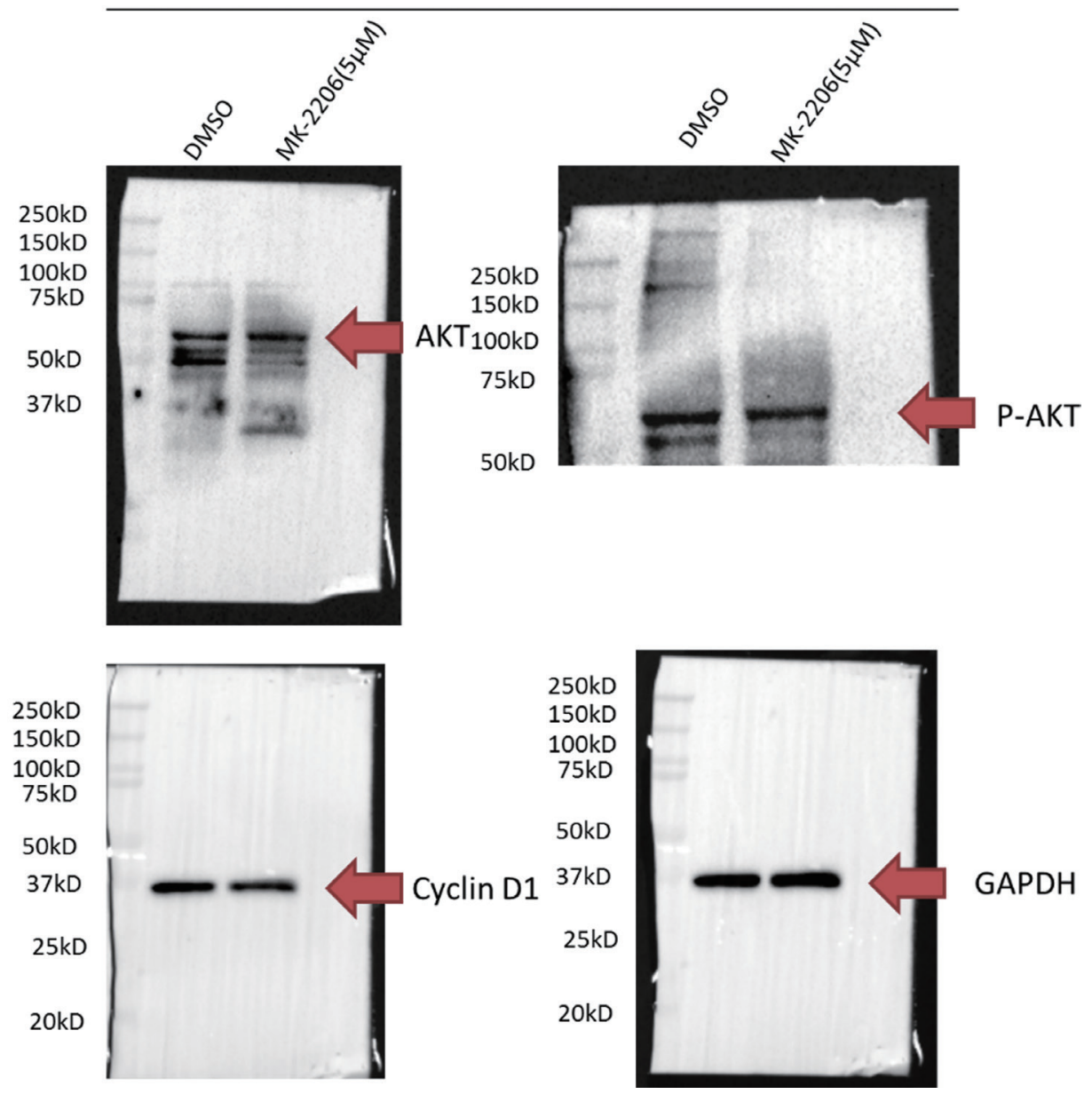

FIGURE 6E

MDA-MB-468 PCDNA3-ESM1

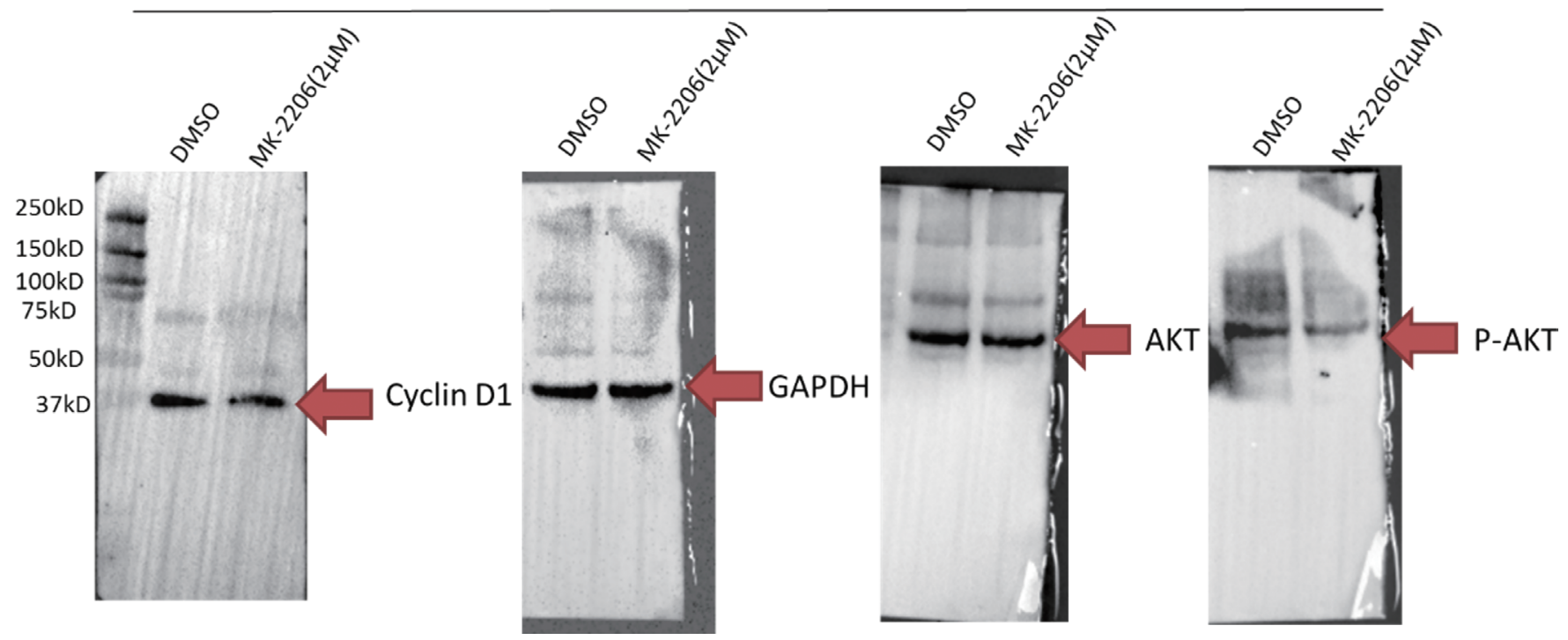




\section{FIGURE 6F}

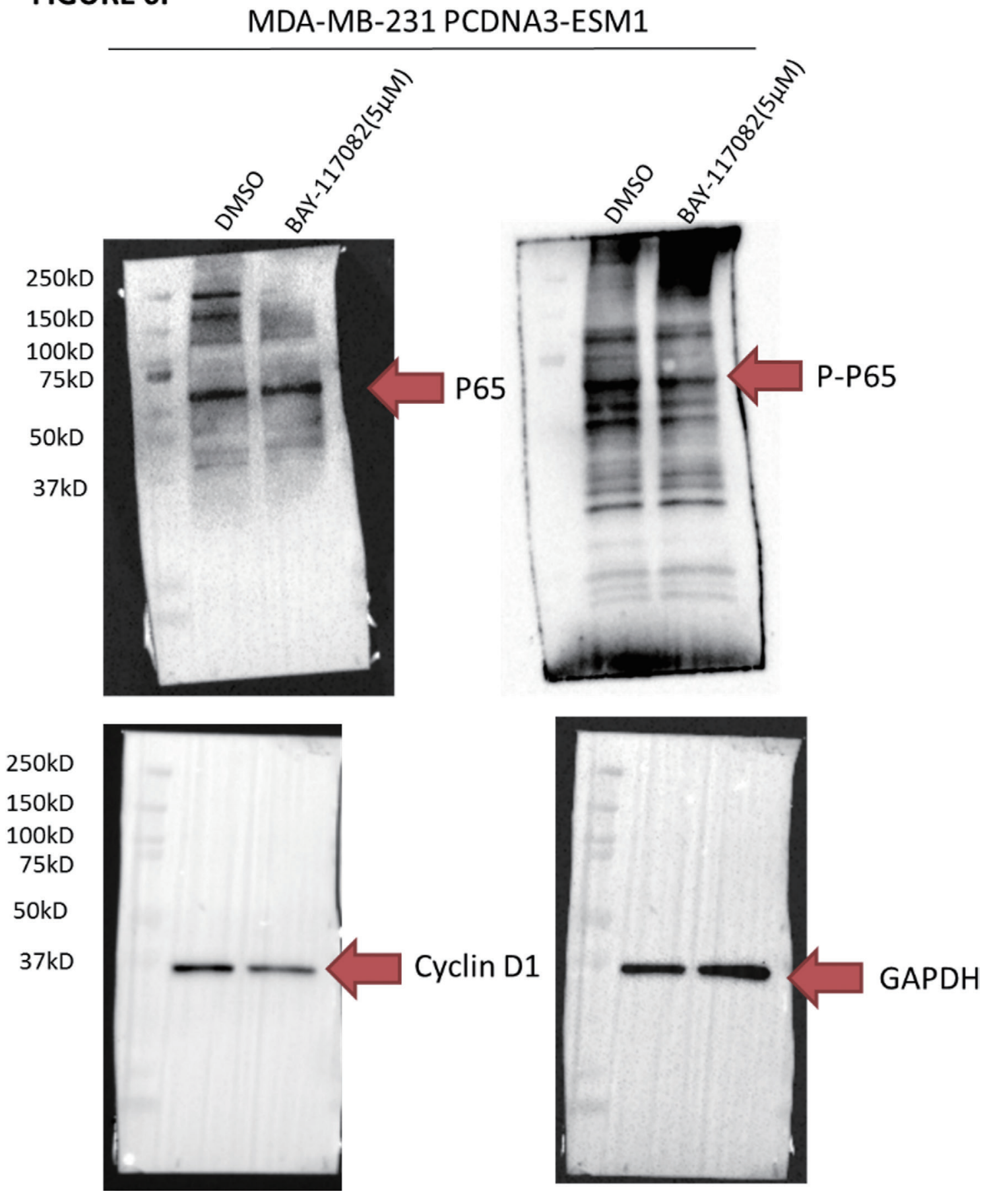




\section{FIGURE 6F}

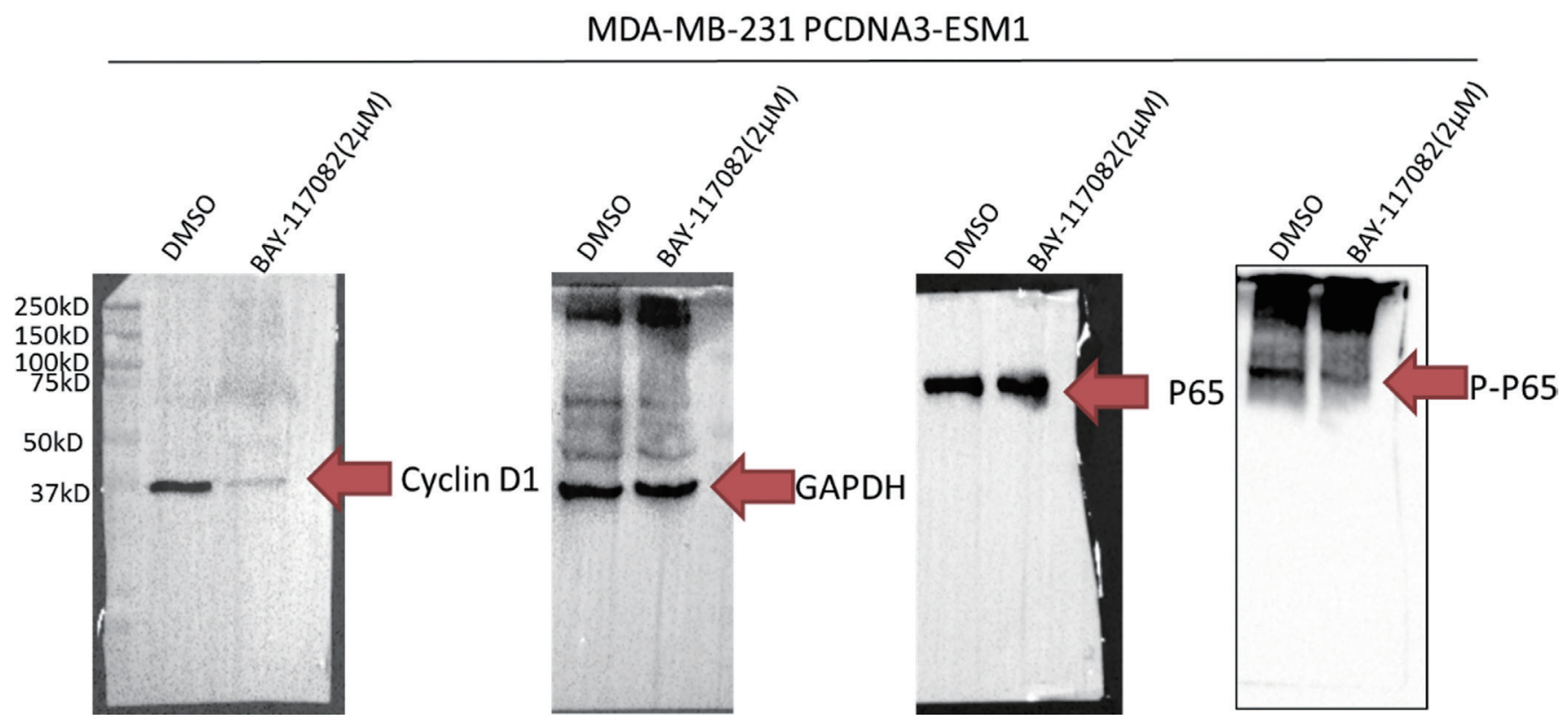

Figure S1 Original blots of the Western blot shown Figures 1,2 and Figures 5,6.

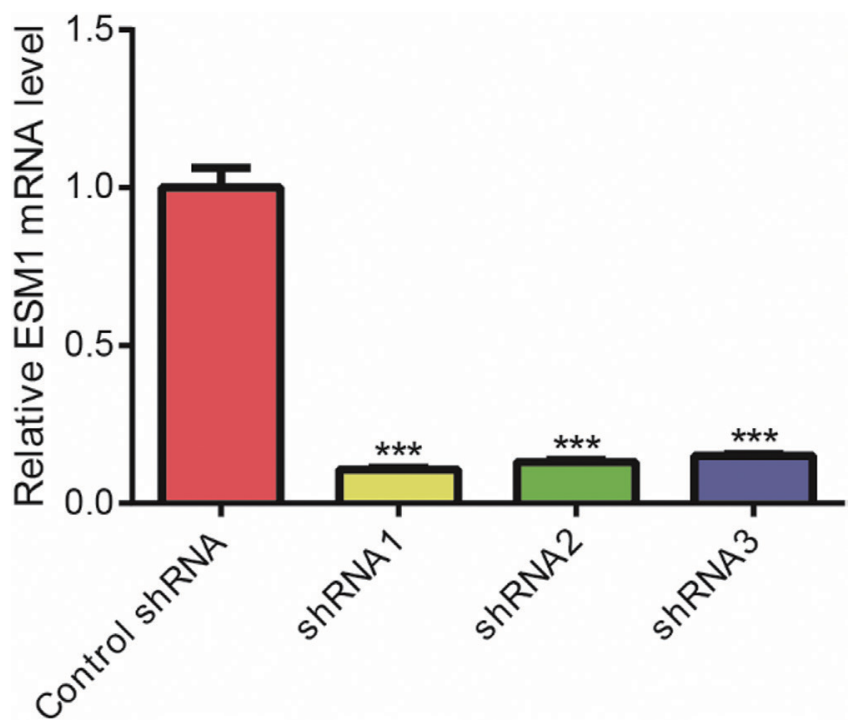

Figure S2 ESM1 mRNA expression in MDA-MB-231 cells was efficiently knocked down by all three shRNAs. ${ }^{* * *}, \mathrm{P}<0.001$. 


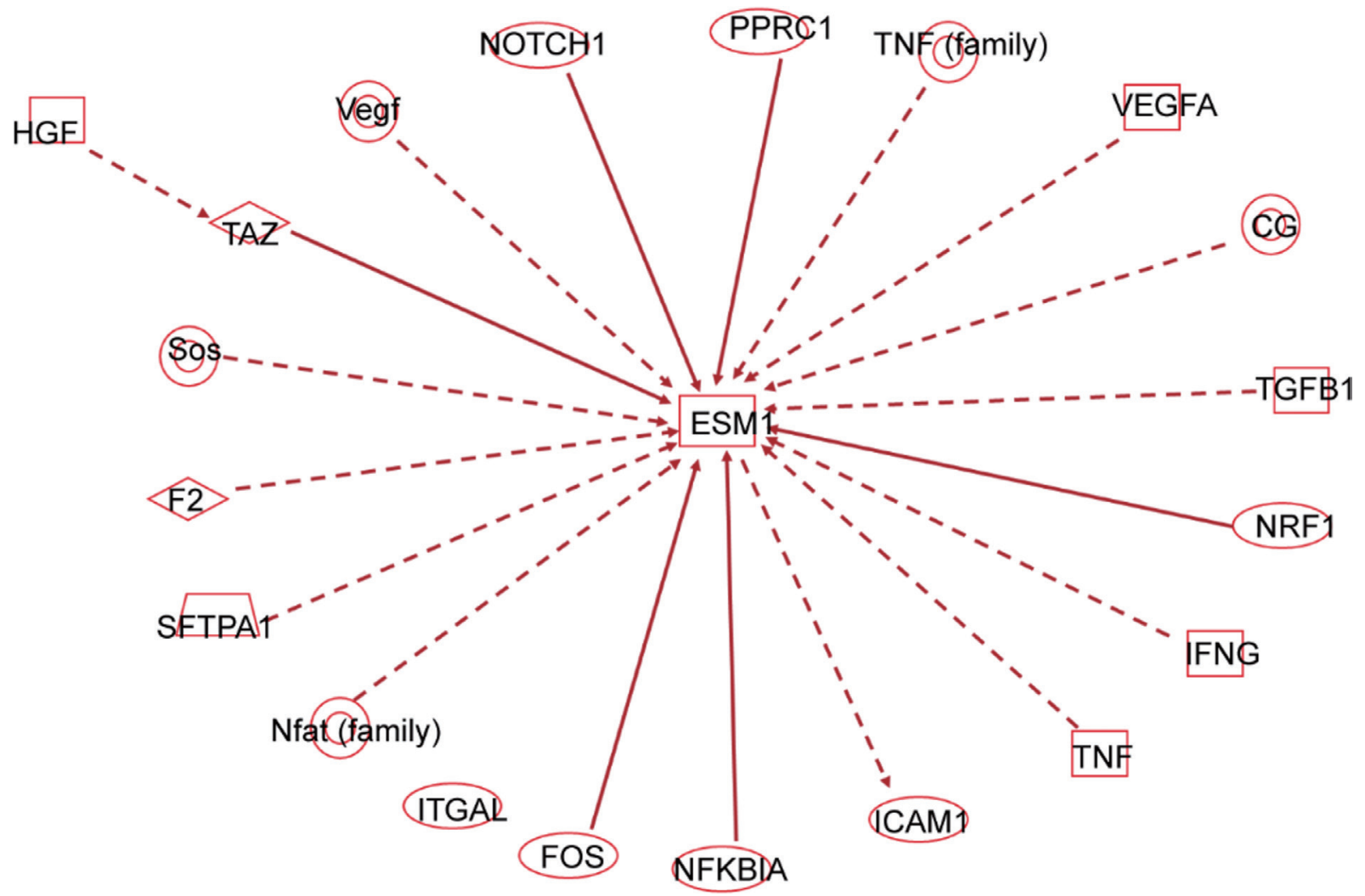

Figure S3 Bioinformatic analysis predicts the regulation network of ESM1. Regulation network of ESM1 was constructed using QIAGEN's Ingenuity ${ }^{\circledR}$ Pathway Analysis tools. 